\title{
Modeling the connection between primary and metastatic tumors
}

\author{
David Diego • Gabriel F. Calvo • \\ Víctor M. Pérez-García
}

Received: 11 January 2012 / Revised: 26 May 2012 / Published online: 25 July 2012

(C) Springer-Verlag 2012

\begin{abstract}
We put forward a model for cancer metastasis as a migration phenomenon between tumor cell populations coexisting and evolving in two different habitats. One of them is a primary tumor and the other one is a secondary or metastatic tumor. The evolution of the different cell phenotype populations in each habitat is described by means of a simple quasispecies model allowing for a cascade of mutations between the different phenotypes in each habitat. The cell migration event is supposed to be unidirectional and take place continuously in time. The possible clinical outcomes of the model depending on the parameter space are analyzed and the effect of the resection of the primary tumor is studied.
\end{abstract}

Keywords Tumor growth · Metastasis - Quasispecies · Mathematical model

\section{Mathematics Subject Classification 92B05 - 92D25}

\section{Introduction}

Metastasis comprises a sequence of linked steps leading to the dissemination of cancer cells from a primary tumor to other distant tissues. From a clinical point of view it

\footnotetext{
D. Diego $(\bowtie)$ • V. M. Pérez-García

Departamento de Matemáticas, E.T.S.I. Industriales

and Instituto de Matemática Aplicada a la Ciencia y la Ingeniería,

Universidad de Castilla-La Mancha, 13071 Ciudad Real, Spain

e-mail: susydiegolas@gmail.com

V. M. Pérez-García

e-mail: victor.perezgarcia@uclm.es

G. F. Calvo

Departamento de Matemáticas, E.T.S.I. Caminos, Canales y Puertos

and Instituto de Matemática Aplicada a la Ciencia y la Ingeniería,

Universidad de Castilla-La Mancha, 13071 Ciudad Real, Spain

e-mail: gabriel.fernandez@uclm.es
} 
is directly associated with a poor prognosis of the disease; despite improvements in surgical techniques, general patient care, and local and systemic adjuvant therapies, the overwhelming majority of cancer-related deaths still result from the progressive growth of metastasis that are resistant to conventional therapies.

In the metastatic cascade, a subpopulation of malignant cells within the primary tumor first acquires the ability to move and degrade the surrounding extracellular matrix and initiate an invasion process. The subsequent events allow migrating cancer cells to penetrate the bloodstream through the blood vessels or via the lymphatic system and settle at distant tissues of the body from the parent neoplasm, thus initiating a secondary tumor. The process of metastasis is an obstacle race that tumor cells have to overcome with different adaptations (Weiss 2000; Chambers et al. 2000, 2001, 2002; Pantel 2004) and has traditionally been considered as a final stage in tumorigenesis (Hanahan and Weinberg 2000), since the ability to metastasize has been thought to be acquired only when the tumor, nurtured by an angiogenic vasculature, reaches a large number of cells. More recently, several lines of evidence suggest that the onset of metastasis could, as well, be an early event in carcinogenesis (Bernards and Weinberg 2002; Chaffer and Weinberg 2011; Hanahan and Weinberg 2011), relating the spread of cancer cells to the alteration of specific oncogenes and tumor suppressor genes (Hüsemann et al. 2008; Podyspanina et al. 2008).

The connection between primary and secondary tumors goes even far beyond the physical scatter/exchange of tumor cells. It is well known that the surgical resection of the primary tumor is followed, in certain cases, by a rapid outgrowth of tumor metastasis (Ebos et al. 2009; Peeters et al. 2004, 2005, 2006, 2008). This phenomenon is thought to be related with the secretion of some metastatic suppressor factors (Cook et al. 2011; Hurst and Welch 2011), although detailed clinical studies are still lacking.

From the mathematical point of view a number of papers have considered, with different levels of sophistication, specific aspects of the metastatic cascade following a variety of perspectives. Already in 1985, Koscielny et al. (1985), using both exponential and Gompertzian growth patterns, studied the natural history of human breast cancer in 2,648 patients, and proposed a very simple model in which metastasis was initiated only after the primary tumor had reached a threshold volume. In another model (Retsky et al. 1997), it was assumed that the growth of breast cancer metastases passed through three sequential phases: an initial dormancy of isolated metastatic cells, a second phase of avascular replication, and a third phase of vascularized growth. This model was used to explain a bimodal relapse distribution from 1,173 cases of untreated early breast cancer and how chemoresistance to adjuvant therapy leads to delayed relapse. More recently, several authors have addressed the computation of the probability for metastatic formation on the basis of stochastic models of tumorigenesis: (Yorke et al. 1993; Kendal 2001; Iwasa et al. 2004; Michaelson et al. 2005; Michor et al. 2006; Traulsen et al. 2006; Dingli et al. 2007; Basanta et al. 2008; Haeno and Michor 2010; Hanin and Korosteleva 2010). The same problem has been approached using individual cell-agent based computational models in which the metastasis progression is derived from the individual dynamics of stem cells and their progeny by employing different tumor migration potentials (Enderling et al. 2009). Finally, energy-statistical models considering the metastatic dissemination to be triggered by an 'energetic crisis' undergone by the cancer cells after surpassing a certain 
primary tumor mass threshold have been recently developed (Dattoli et al. 2009). Other models have instead dealt with tumor dissemination to surrounding tissues in terms of spatial models (Gatenby and Gawlinski 1996; Boushaba et al. 2006; Frieboes et al. 2006; Jain et al. 2007; Gerisch and Chaplain 2008; Ramis-Conde et al. 2008; Bearer et al. 2009; Eikenberry et al. 2009; Andasari et al. 2010). Finally, the computation of the topographical patterns of metastasis development has also been considered using network models (Chen et al. 2009).

All of the above mentioned approaches have proven to be useful descriptions of different aspects of metastasis progression, although, one important difficulty present in most of these models is the large number of parameters required and the limited empirical information for their assessment. Besides, to the best of our knowledge, only few works have been devoted to study the communication between primary and metastatic tumors. Any realistic mathematical model of metastasis should reflect the cellular phenotype and genotype heterogeneity encountered within the developing ectopic neoplastic tissues. Taking all these considerations into account, in this paper we address the issue of communication between primary and metastatic tumors from the dynamical evolution of several tumor phenotypes where all cell populations are governed by a system of Lotka-Volterra-like equations and investigate the possible clinical outcomes depending on the parameter space. For the sake of clarity, the paper follows a bottom-up approach, that is: firstly, we establish the main aspects to be addressed as well as the capability of our approach to model them by an analytical study of a reduced version. The number of parameters remains small and their biological meaning is thoroughly discussed. Once the main features predicted by the simplest model are well understood we proceed to increase the complexity of the model by incorporating a hierarchy in the way cell phenotypes emerge, interact and disappear. As we shall see, the basic features of the simple model are preserved and enriched within the more complicated ones.

The paper is organized as follows: First, in Sect. 2 we present a simple mathematical model for metastasis consisting of two habitats with two cell subpopulations in each of them: tumor and normal cells. By studying the phase plane we shall identify some interesting phenomena related with the connection between the primary tumor and the metastatic habitat as well as the effect on the latter after the resection of the primary tumor. In Sect. 3 we extend the model to incorporate an arbitrarily large number of tumor cell phenotypes, normal cells and necrotic tissue. By restricting the extended model to few tumor phenotypes we will numerically check the solutions and find the same basic features already encountered in the previous simpler scenario. The conclusions and future perspectives are discussed in Sect. 4. For completeness, the structure of the steady state solutions of the extended model as well as some other technical aspects are addressed in Appendix 4.

\section{Mathematical model for coupled primary and metastatic tumors evolution}

To illustrate our ideas we begin with a simplified model describing the evolution of a population of tumor cells coexisting with normal (healthy and well differentiated) cells within two distant (yet weakly connected) habitats: a primary tumor and a secondary 
or metastatic tumor. The primary habitat is taken as the one where the tumor originates and the malignant cells therein present may eventually disseminate with a rather small migration rate toward a secondary tissue thus resulting in a metastatic tumor. Possible reseeding of the primary tumor by its derived metastasis, although experimentally observed in mouse models of breast carcinoma, colon carcinoma and malignant melanoma (Kim et al. 2009), is thought to be a rare event and will not be included for simplicity.

\subsection{Evolution equations}

The equations presented below are meant to dictate the evolution of local densities of cell populations rather than the total number of them. This justifies the concept of a maximum cell density that a given tissue can accommodate, say $\rho_{\mathrm{P}, \max }$ for the primary tissue and $\rho_{\mathrm{S} \text {, max }}$ for the secondary one. In the non-pathological scenario, these correspond to the normal cell population densities for each specific tissue. However, it should be stressed that in some tumor types (e.g., small-cell lung carcinomas usually leading to brain metastases Hayasida et al. 2006), the maximum cell densities can achieve significantly higher values than those of the original normal tissue, say $\rho_{\mathrm{P}, \mathrm{T}} \geq \rho_{\mathrm{P}, \max }$ for the primary habitat and $\rho_{\mathrm{S}, \mathrm{T}} \geq \rho_{\mathrm{S}, \max }$ for the secondary habitat, respectively. Henceforth, rather than determining the absolute cell densities, we will find more revealing, for describing the many possible outcomes within the primary and secondary habitats, to consider instead the ratio between the distinct spatially averaged tumor cell phenotype densities over the invaded organ and the average maximal normal cell density in premalignant conditions, that is: $P_{i} \rightarrow P_{i} / \rho_{P, \max }$ and $S_{i} \rightarrow S_{i} / \rho_{S \text {, max }}$, with $P_{i}\left(S_{i}\right)$ representing any of the cell populations within the primary (secondary) habitat.

In the primary habitat, $P_{T}$ will refer to the population density ratio corresponding to the malignant cells, while $P_{H}$ will stand for the normal cells. Similarly, for the secondary habitat, $S_{T}$ will represent the malignant cells whereas the healthy population will be labeled as $S_{H}$. Hereafter, it will be understood that all population densities are nonnegative quantities.

The proposed set of equations governing the interacting populations is given by the following autonomous first-order system

$$
\begin{aligned}
& \dot{P}_{T}=\Gamma_{T}\left(\Psi-P_{T}-P_{H}\right) P_{T}-\Delta_{T} P_{T}-k P_{T}, \\
& \dot{P}_{H}=\Gamma_{H}\left(1-P_{T}-P_{H}\right) P_{H}-\Delta_{H} P_{T} P_{H}, \\
& \dot{S}_{T}=\gamma_{T}\left(\psi-S_{T}-S_{H}\right) S_{T}-\delta_{T} S_{T}-\epsilon P_{T} S_{T}+\tilde{k} P_{T}, \\
& \dot{S}_{H}=\gamma_{H}\left(1-S_{T}-S_{H}\right) S_{H}-\delta_{H} S_{T} S_{H} .
\end{aligned}
$$

In general, we will use upper case greek letters to refer to the parameters of the primary habitat while lower case letters will refer to parameters of the secondary habitat. In Eq. (1), $\Gamma_{T}, \Gamma_{H}, \gamma_{T}$ and $\gamma_{H}$ denote the different proliferation rates while $\Delta_{T}, \Delta_{H}, \delta_{T}$ and $\delta_{H}$ are the death rates, all of them measured in units of inverse of time. The first terms in the right-hand-side of Eq. (1) are the usual logistic forms describing the effect 
of population saturation due to limited cell density capacity. The normalized carrying capacity values $\Psi \equiv \rho_{\mathrm{P}, \mathrm{T}} / \rho_{\mathrm{P}, \max }$ and $\psi \equiv \rho_{\mathrm{S}, \mathrm{T}} / \rho_{\mathrm{S}, \max }$ account for the ratio of malignant relative to the normal maximum cell densities.

An essential ingredient in our model is the presence of the terms $-k P_{T}(t)$ and $\tilde{k} P_{T}(t)$ in Eq. (1a) and (1c), respectively. They represent cell emigration (from the primary tumor) and immigration (invasion of the secondary tissue) and are taken to depend on the instantaneous density of malignant cells in the primary tumor at time $t$. A more accurate immigration term should include the average time $\tau$ of physical translocation of the circulating tumor cells from the primary to the secondary site. Thus, one would expect a delayed term of the form $\tilde{k} P_{T}(t-\tau)$. However, we will be interested in examining the global evolution of the primary and secondary tumors for time scales much longer than $\tau$ (days to weeks). For instance, $\tau$ is of the order of $1-2.4 \mathrm{~h}$ in the case of patients with breast cancer dormancy (Meng 2004), and so we may safely neglect this delayed effect. The constants $k$ and $\tilde{k}$ are the corresponding emigration and immigration (homing) rates, respectively. On a physiological basis, one may assume that $0 \leq \tilde{k} \leq k$. Whether a minute or a significant fraction of the cells released from the primary tumor will actually reach the secondary tissue (the parenchyma) remains to be fully understood, although it is suspected to be an inefficient process in the sense that a non-negligible fraction of tumor cells die during the course of migration. It is known that cell spread to the anatomically distant sites appears to take place mainly through the blood vessels via the process of hematogenous dissemination (Chambers et al. 2002; Chaffer and Weinberg 2011), while lymphogenous metastasis (involving the lymph nodes) would represent a dead end. Many patients with advanced primary carcinomas contain circulating tumor cells in their blood samples; so it is expected that at least a subset of these may be in transit from the primary tumor to other tissues for colonization. For circulating tumor cells to be able to colonize distant sites they must develop, among others, the capability to evade the immune system response and resist the sieving action of the microvasculature when their diameters $(20-30 \mu \mathrm{m})$ are larger than the typical size of capillaries $(\sim 8 \mu \mathrm{m})$. Whether these (and other) mechanisms act as a phenotype selection driving force once the tumor cells are in the circulatory system or, else, that immune avoidance and cell plasticity are traits acquired in the primary tumor remain also unclear. Within our model, $\tilde{k} \ll k$ would be associated with the former selection mechanism whereas $\tilde{k} \lesssim k$ would correspond to the latter one. In our subsequent analysis we will retain the term $-k P_{T}$ although one would expect that the emigration rate $k$ satisfies $k \ll \Delta_{T}$, as the fraction per unit time of tumor cells shed to the vasculature is much smaller than the tumor cells undergoing apoptosis/necrosis.

The Eq. (1c) includes the term $-\epsilon P_{T} S_{T}$ which exhibits a dependence on the (instantaneous) malignant population at the primary tumor that modulates the growth of the metastasis. In some types of cancer, such as breast cancer and melanoma, it has been observed that upon surgical resection of the primary tumor, secondary distant tumors can display an explosive growth soon after the removal of the primary tumor or, else, render dormant such micrometastases for decades (Folkman 2002; Demicheli et al. 2008). This process is thought to be related with the release of metastasis suppressors. These are defined as molecules whose expression results in the inhibition of a neoplastic cell ability to metastasize while having little effect on primary tumor growth 
(Cook et al. 2011; Hurst and Welch 2011). As a mater of fact, over thirty metastasis suppressors have already been identified although the precise mechanisms of action for most of them are still unknown. These molecules are found within cells and in the extracellular melieu and a subset of them are known to inhibit the growth of tumor cells after they have already disseminated. Finally, some of the metastasis suppressors are secreted by the tumor cells and it is hypothesized that the interplay with the stromal population could induce the tumor growth inhibition (Cook et al. 2011).

In the context of our model, we will describe this suppression effect by assuming that the metastatic growth is down-regulated by the interplay between the secretion of metastasis suppressors from both the primary and the metastatic tumors and, for the sake of simplicity of the model, we choose the above quadratic term, where $\epsilon$ is a positive quantity standing for an effective suppression rate.

Finally, such suppression effect has not been included in the evolution equation for the normal cells, Eq. (1d), since the tumor cells are strongly dependent upon genetic alterations that distinguish them from their well differentiated normal counterparts (for instance, showing a hyper sensitivity to extracellular factors that may, or may not, allow them to metastasize successfully Cook et al. 2011) and as a consequence, we expect the suppression effect to be marginal on them.

The issues of chemical communication between primary and secondary tumors and the effect of the resection of the primary tumor has been addressed in few previous works. For example, a spatial model described the spread of growth inhibitory factors secreted by the primary tumor and specialized on the development of polypoid melanoma (Boushaba et al. 2006). The model predicted that if metastases are close enough to the primary tumor $(d \lesssim 5.5 \mathrm{~cm})$ they will become extinct regardless of the removal of the former. However, if the metastases are located at a moderate distance $(5.5 \lesssim d \lesssim 8.5 \mathrm{~cm}$ ) the removal of the primary tumor induces the growth of those metastases while they become extinct if the primary tumor is not resected. If, on the other hand, the metastatic tumor is far enough $(d \gtrsim 50 \mathrm{~cm})$ its growth is independent of the primary tumor.

Eikenberry et al. (2009) proposed an exhaustive diffusion-reaction model for tumorimmune system interaction applied to malignant melanoma. The authors suggested that the aggressive metastasis growth following surgical excision of the primary tumor could be understood within such interaction. The reaction of the immune system to the presence of the primary tumor may suppress nearby metastases whereas after the surgical resection most of the immune cells are removed and the previously checked local metastases may resume their aggressive growth.

Our model also addresses the communication between the primary and metastatic tumors, although using a simpler non spatial and semi-analytical model with various malignant phenotypes. Yet we find, as will be shown below, non trivial features related with the communication between both habitats, such as: a window for growth boost of the secondary tumor after the resection of the primary tumor by means of the presence of the term $-\epsilon P_{T} S_{T}$ which weakly connects the metastatic and the primary sites. An important difference is that within our model both the relative carrying capacity of the secondary tumor, $\psi$, and the effective suppression rate, $\epsilon$, play a key role in the interaction between both habitats. Finally, the death terms for the normal cells in Eq. (1b) and (1d) take into account that the surrounding tumor cells, by releasing metabolites 
to the microenvironment (e.g. secretion of lactate contributing to the acidification of the microenvironment), may promote or trigger apoptosis (Cairns et al. 2011).

Since the primary habitat is where the tumor originates, we will assume that a small initial fraction of malignant cells arises embedded in a tissue of normal cells. Within the secondary habitat, however, we will not regard any genetic alteration of normal cells; the only source of malignant cells will be those ectopically settling from the circulating ones. Accordingly, the initial value will be taken to be $S_{H}(0)=1, S_{T}(0)=0$.

\subsection{Steady-state solutions}

The possible steady-state solutions of our model equations (1a)-(1d) and their stability will be analyzed by resorting to the $P_{T}-P_{H}$ and $S_{T}-S_{H}$ phase planes.

\subsubsection{Primary habitat}

Note that the primary habitat is essentially uncoupled from the secondary one, thus it constitutes a second order autonomous dynamical system. The set of points on the positive quadrant $\left(P_{H}, P_{T}>0\right)$ of the $P_{T}-P_{H}$ plane at which $\dot{P}_{H}$ and $\dot{P}_{T}$ respectively vanish verify the equations

$$
\begin{gathered}
\dot{P}_{T}=0 \rightarrow\left\{\begin{array}{l}
P_{T}=0 \\
P_{H}=\Psi-\frac{k+\Delta_{T}}{\Gamma_{T}}-P_{T}
\end{array}\right. \\
\dot{P}_{H}=0 \rightarrow\left\{\begin{array}{l}
P_{H}=0 \\
P_{H}=1-\frac{\Gamma_{H}+\Delta_{H}}{\Gamma_{H}} P_{T}
\end{array}\right.
\end{gathered}
$$

Since we assume that $0 \leq P_{H} \leq 1$ then $0 \leq P_{T} \leq \Psi-\left(k+\Delta_{T}\right) / \Gamma_{T}$. Figure 1 shows the $P_{T}-P_{H}$ plane for the populations in the primary habitat together with the regions of increasing and decreasing populations. In the following plots (and all the plots throughout this section) the dashed lines will represent the characteristics in Eq. (2a) and (4a) while the dashed-dotted lines will account for the set of points of vanishing time derivative of the normal population given by Eq. (2b) and (4b). On the other hand, solid lines will account for the different integral curves when solving Eq. (1). Notice that, for the primary habitat, above the vanishing derivative lines given in Eq. (2a)-(2b) both population densities decrease whereas below those lines the population densities increase with time.

Accordingly, three possible regimes arise depending on the values of the quantity $\Psi-\frac{k+\Delta_{T}}{\Gamma_{T}}$. In what follows we examine all three of them:

First, when $\Psi-\frac{k+\Delta_{T}}{\Gamma_{T}} \geq 1$ the straight curve of vanishing tumor density derivative (dashed line) remains above that corresponding to the vanishing of the growth of normal cells (dashed-dotted line) and as a consequence there are only two possible fixed points for the dominance of either the tumor $\left(P_{T}^{*}=\Psi-\frac{k+\Delta_{T}}{\Gamma_{T}}, P_{H}^{*}=0\right)$ or the normal $\left(P_{T}^{*}=0, P_{H}^{*}=1\right)$ cells, respectively. 

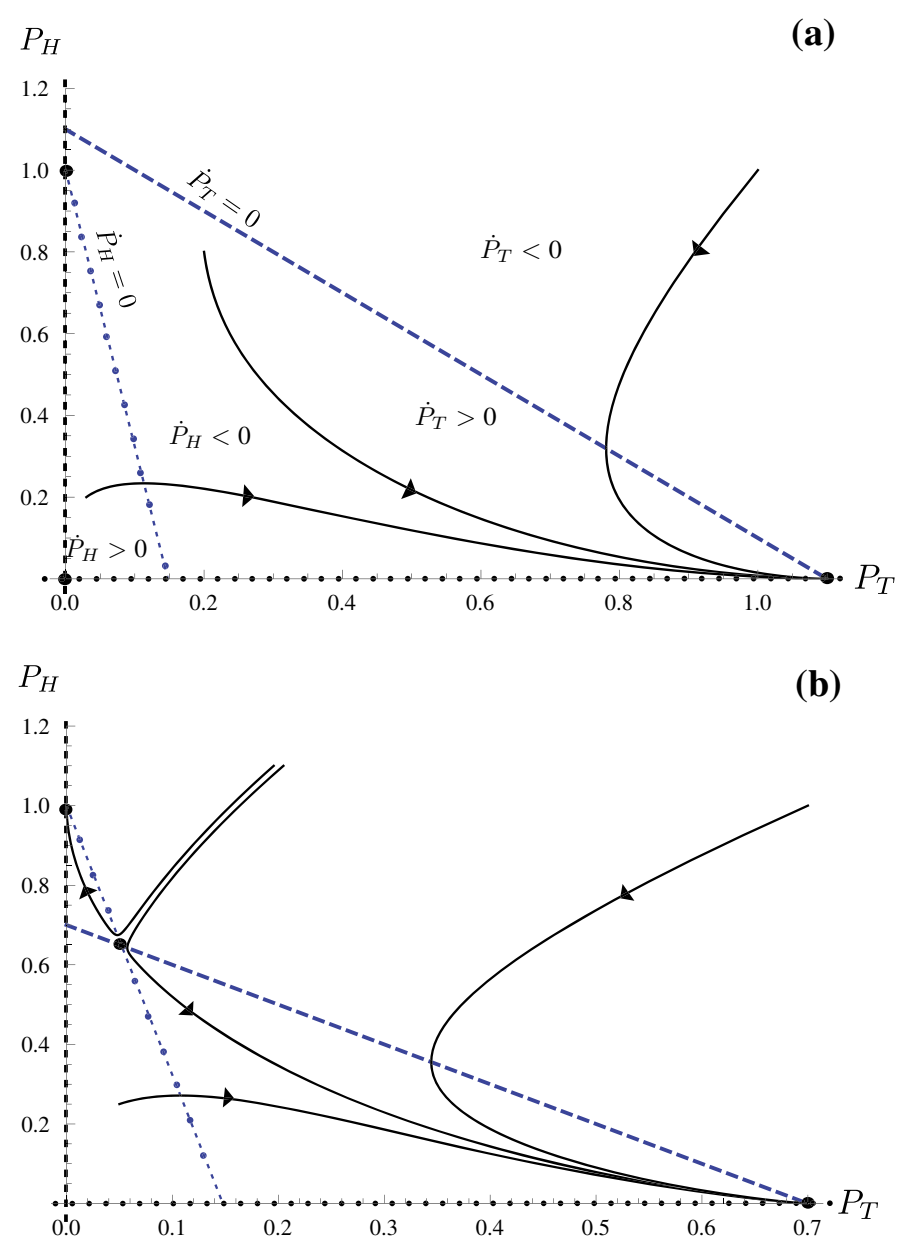

Fig. $1 P_{T}-P_{H}$ phase plane for $\mathbf{a} P_{T}^{*}=1.1$ and $\mathbf{b} P_{T}^{*}=0.7$. The dashed lines correspond to the phase space points where $\dot{P}_{T}=0$ while the dashed-dotted lines stand for those points where $\dot{P}_{H}=0$. The solid lines represent examples of different integral curves

Within the region between the vanishing derivative curves, the tumor population increases while the normal density decreases. Furthermore, any integral curve starting within such region will not cross any of these lines, and therefore the tumor fixed point is the only possible steady state for all of them. This makes the fixed point for only normal cells unstable. On the other hand, any integral curve close enough to the tumor fixed point will eventually approach this steady state and, as a consequence, the tumor fixed point is stable.

A second regime corresponds to

$$
\frac{\Gamma_{H}}{\Gamma_{H}+\Delta_{H}}<\Psi-\frac{k+\Delta_{T}}{\Gamma_{T}}<1,
$$

in which case a third fixed point arises for which both $P_{T}^{*}$ and $P_{H}^{*}$ are non zero. 
Table 1 Stability of the critical points for the different parameter regimes as a function of $\Psi$ in the primary habitat

\begin{tabular}{lllll}
\hline Parameter region & Trivial & Tumor cells only & Normal cells only & Coexistence \\
\hline$\Psi-\frac{k+\Delta_{T}}{\Gamma_{T}} \geq 1$ & $\mathrm{U}$ & $\mathrm{S}$ & $\mathrm{U}$ & $\mathrm{N}$ \\
$\frac{\Gamma_{H}}{\Gamma_{H}+\Delta_{H}}<\Psi-\frac{k+\Delta_{T}}{\Gamma_{T}}<1$ & $\mathrm{U}$ & $\mathrm{S}$ & $\mathrm{S}$ & $\mathrm{U}$ \\
$0<\Psi-\frac{k+\Delta_{T}}{\Gamma_{T}} \leq \frac{\Gamma_{H}}{\Gamma_{H}+\Delta_{H}}$ & $\mathrm{U}$ & $\mathrm{U}$ & $\mathrm{S}$ & $\mathrm{N}$ \\
\hline
\end{tabular}

$N, S$ and $U$ stands for none, stable and unstable, respectively

The same reasoning as before shows that the new coexisting equilibrium is an unstable fixed point, while the only malignant and the only normal cells fixed points are both stable.

Finally, a third regime corresponding to $0<\Psi-\frac{k+\Delta_{T}}{\Gamma_{T}} \leq \frac{\Gamma_{H}}{\Gamma_{H}+\Delta_{H}}$, leads again only to malignant or normal equilibria, although in this case the disposition of vanishing population derivatives is exactly the opposite to the first case. Thus, the tumor fixed point is unstable while the normal cells equilibrium turns out to be stable.

Table 1 summarizes the previous discussion and contains all of the possible steady solutions together with their stability.

In what follows we will assume that $\Psi>1$, reflecting the fact that tumor cells possess an increased advantage with respect to the normal cells to proliferate in the primary habitat. Regarding the carrying capacity $\psi$ of the tumor cell phenotype in the secondary habitat, we will consider a more general situation in which either $\psi>1$ or else $0<\psi \leq 1$; the invading tumor cells may or may not exhibit a survival advantage in the secondary habitat.

\subsubsection{Secondary habitat}

Although the secondary habitat is not governed by an autonomous system, in the steady state its behavior depends only on the equilibria of the populations in the primary tumor. In general, for a given value of $P_{T}$, namely, a time slice, the curves of vanishing normal and tumor population derivatives (out of the $S_{T}$ axis) are given by

$$
\begin{aligned}
& S_{H}=\psi-\frac{\epsilon}{\gamma_{T}} P_{T}-\frac{\delta_{T}}{\gamma_{T}}+\frac{\tilde{k}}{\gamma_{T}} \frac{P_{T}}{S_{T}}-S_{T}, \quad\left(\dot{S}_{T}=0\right) . \\
& S_{H}=1-\frac{\gamma_{H}+\delta_{H}}{\gamma_{H}} S_{T}, \quad\left(\dot{S}_{H}=0\right),
\end{aligned}
$$

One can easily show that these two curves intersect at nonnegative values of $S_{T}$ and $S_{H}$ if, and only if,

$$
f_{\min } \equiv 2 \sqrt{\frac{\delta_{H} \tilde{k} P_{T}}{\gamma_{T} \gamma_{H}}}-\frac{\delta_{T}}{\gamma_{T}}-\frac{\epsilon}{\gamma_{T}} P_{T}+\psi-1 \leq 0,
$$




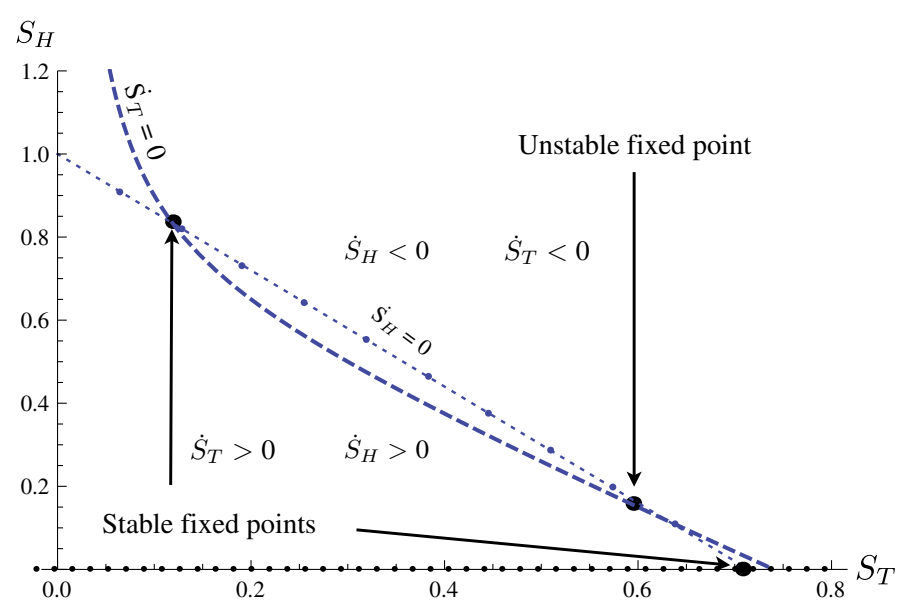

Fig. 2 Fixed points and their stability for the secondary habitat. We depict the phase plane for parameter values: $P_{T}^{*}=1.2, \delta_{H} / \gamma_{H}=0.4, \delta_{T} / \gamma_{T}=0.2, \tilde{k} / \gamma_{T}=0.025, \epsilon \simeq 0.054 \mathrm{day}^{-1}$ and $\psi=1.02$

presenting a unique intersection when the equality holds, and crossing twice whenever the above quantity is strictly negative. Notice that in the secondary habitat the stability of the solutions follows a different pattern from that of the primary one. In particular, if there is a coexistence solution, the first of the point cuts, that is, the one with the lowest malignant component, is always a stable fixed point. Unlike in the primary habitat, before the first coexistence solution, the curve of vanishing malignant population derivative lies above that corresponding to the normal population and otherwise after the first intersection. The signs of the derivatives then dictate that around such solution all the integral curves will eventually hit the corresponding fixed point as indicated in Fig. 2.

Figure 3 schematically shows the different behaviors, within the secondary habitat, depending on the sign of $f_{\min }$. In both Figs. 2 and 3 the particular choice of the data has been made for the sake of clarity of the graphics and is not intended to be a biologically accurate choice. The two roots of $f_{\min }=0$ for $\sqrt{P_{T}}$ are given by

$$
\sqrt{R_{ \pm}}=\sqrt{\frac{\delta_{H} \gamma_{T} \tilde{k}}{\epsilon^{2} \gamma_{H}}} \pm \sqrt{\frac{\delta_{H} \gamma_{T} \tilde{k}}{\epsilon^{2} \gamma_{H}}+\frac{\gamma_{T}}{\epsilon}\left(\psi-1-\frac{\delta_{T}}{\gamma_{T}}\right)} .
$$

Now if $P_{T}^{*} \in\left(R_{-}, R_{+}\right)$the population of normal cells within the secondary habitat is annihilated. If otherwise $P_{T}^{*} \leq R_{-}$or $P_{T}^{*} \geq R_{+}$the populations will reach a coexistence equilibrium. The transition from positive to negative values of $f_{\min }$, however, deserves a further comment. Unlike the reverse transition, it might happen that some $\left(S_{T}, S_{H}\right)$ integral curves evolve fast enough to surpass the values of the malignant components corresponding to the intersection points before $P_{T}(t)$ reaches the value $R_{+}$(i.e., before rendering $f_{\min }$ negative), thus asymptotically approaching the extinction of normal cells. However, our numerical simulations show that the evolution within the primary habitat is in general much faster than that in the secondary habitat, as a consequence this path to the extinction of the population of normal cells 


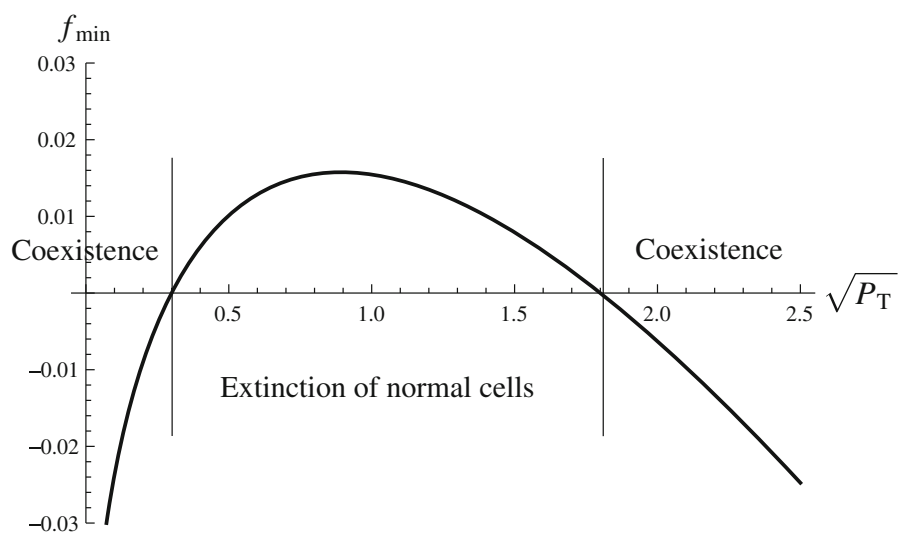

Fig. $3 f_{\min }$ as a function of $\sqrt{P_{T}}$ for a particular choice of parameter values: $\frac{\delta_{T}}{\gamma_{T}}=0.2, \frac{\delta_{H}}{\gamma_{H}}=0.4$, $\frac{\tilde{k}}{\gamma_{T}}=0.02, \psi=1.13$ and $\epsilon \simeq 0.054$ day $^{-1}$

is quickly blocked. This fact sets a threshold for the carrying capacity value $\psi$ in the secondary habitat, given a value of the steady state $P_{T}^{*}$,

$$
\psi_{c}=\frac{\epsilon}{\gamma_{T}}\left(\sqrt{P_{T}^{*}}-\sqrt{\frac{\delta_{H} \gamma_{T} \tilde{k}}{\gamma_{H} \epsilon^{2}}}\right)^{2}-\frac{\delta_{H} \tilde{k}}{\gamma_{H} \epsilon}+\frac{\delta_{T}}{\gamma_{T}}+1,
$$

such that if $\psi \geq \psi_{c}$ the normal cell population disappears, whereas for lower values, the steady state turns into an equilibrium between normal and tumor cell populations. Figure 4 displays the behavior of the populations in the secondary habitat above and below the threshold for a primary steady value $P_{T}^{*}=1.1$ and threshold value for the secondary carrying capacity $\psi_{c} \simeq 1.13$.

Figure 4 a depicts the process of complete metastatic invasion of the secondary habitat. Figure $4 \mathrm{~b}$ shows a dormant metastatic tumor, that is: a coexisting equilibrium in which the fraction of tumor cells is overwhelmed by that of normal cells.

\subsection{Resection}

Let us now consider the effect of the resection of the primary tumor on the dynamics in the secondary habitat. If $\psi>\psi_{c}$ the action of the resection is trivial in the sense that it does not change the structure of the equilibria since the normal cells within the secondary habitat become extinct in both scenarios. However, as we shall see, if $\psi<\psi_{c}$ there is a window of values for the carrying capacity in the secondary habitat in which the resection of the primary tumor triggers the full metastatic invasion within the secondary habitat. Specifically, the secondary tumor steady state density without the presence of the primary tumor is given by

$$
S_{T}^{*}=\frac{\gamma_{H}}{\delta_{H}}\left(1+\frac{\delta_{T}}{\gamma_{T}}-\psi\right)
$$



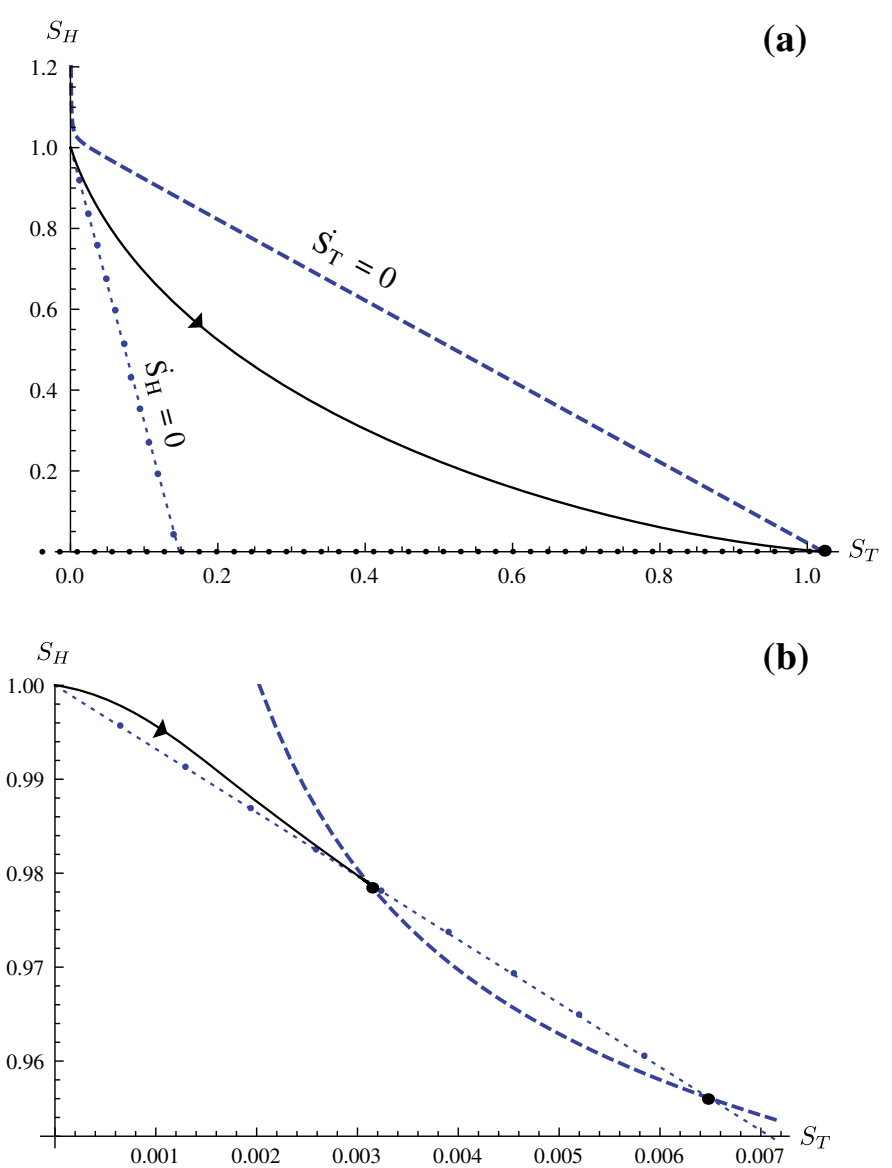

Fig. $4 S_{T}-S_{H}$ phase plane corresponding to $\mathbf{a} \psi \simeq 1.2$ and $\mathbf{b} \psi \simeq 1$.12. In both cases the threshold value for the carrying capacity of tumor cells in the secondary habitat is $\psi_{c} \simeq 1$.13. The solid lines represent integral curves. Note the scales in $(\mathbf{b})$. In both simulations $\epsilon \simeq 0.054$ day $^{-1}$

while, whenever the primary tumor is present, the secondary tumor density is

$$
\tilde{S}_{T}^{*}=\frac{\gamma_{H}}{2 \delta_{H}}\left[1+\frac{\delta_{T}}{\gamma_{T}}+\frac{\epsilon}{\gamma_{T}} P_{T}^{*}-\psi\right]-\sqrt{\frac{\gamma_{H}^{2}}{4 \delta_{H}^{2}}\left[1+\frac{\delta_{T}}{\gamma_{T}}+\frac{\epsilon}{\gamma_{T}} P_{T}^{*}-\psi\right]^{2}-\frac{\gamma_{H} \tilde{k} P_{T}^{*}}{\gamma_{T} \delta_{H}}} .
$$

Now, if $\tilde{S}_{T}^{*}<S_{T}^{*}$, after the resection of the primary tumor the integral curve corresponding to the secondary habitat lies entirely on the region of decreasing tumor population, hence inducing the extinction of the secondary malignant population. If otherwise $\tilde{S}_{T}^{*}>S_{T}^{*}$, once the interaction with the primary habitat has been switched off, the point $\left(\tilde{S}_{T}^{*}, S_{H}^{*}\left(\tilde{S}_{T}^{*}\right)\right)$ belongs to the region of increasing tumor population. Thus, a late resection triggers a metastatic cascade within the secondary tissue, where 

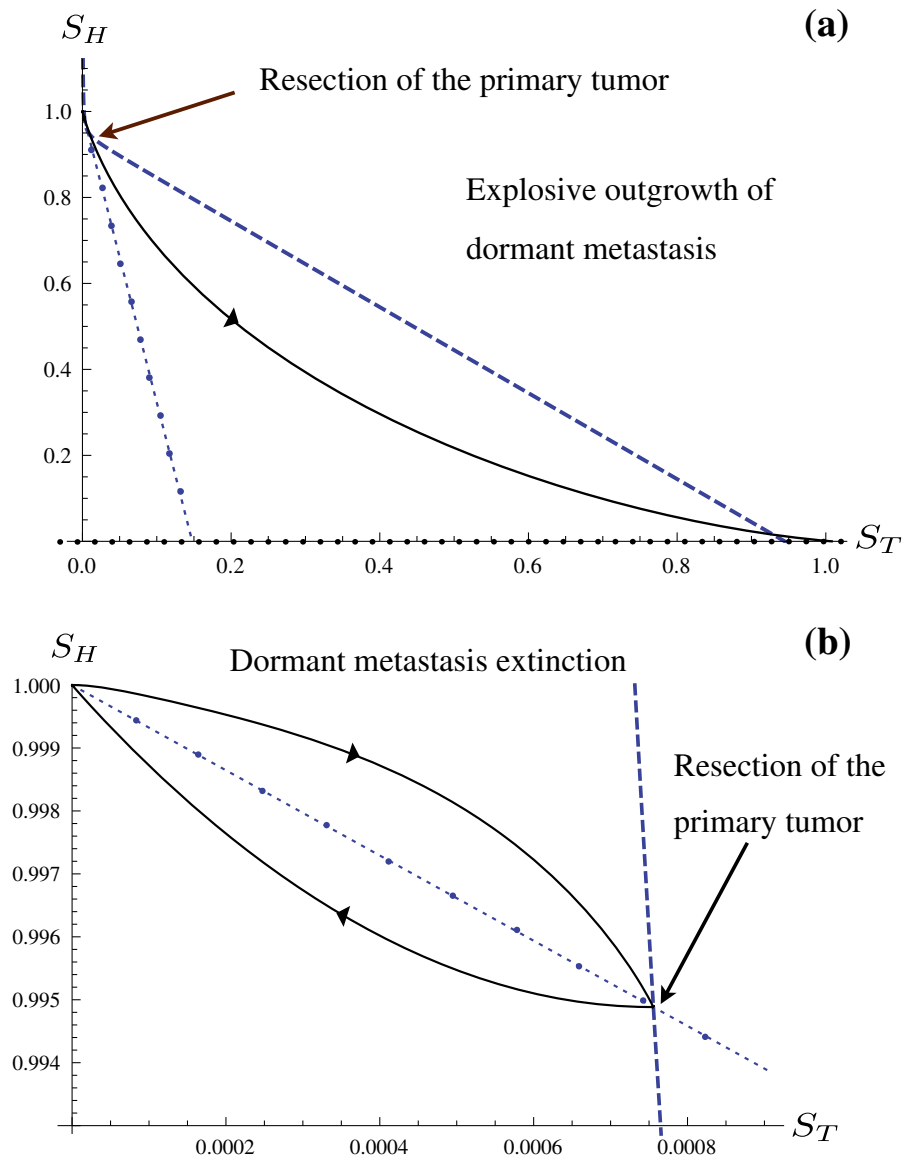

Fig. $5 S_{T}-S_{H}$ phase plane. a Phase plane for $\psi \simeq 1.12$ showing the extinction of normal cells induced by the resection of the primary tumor. b Phase plane for $\psi \simeq 1.02$ showing the tumor extinction within the secondary habitat after the resection of the primary tumor. In both cases the resection of the primary tumor is considered to occur 100 days after the emergence of the malignant cells in the primary habitat. Both cases correspond to a steady primary tumor value of $P_{T}^{*}=1.1$ and $\epsilon \simeq 0.054$ day $^{-1}$

the earliness or lateness of the resection is referred to the time the secondary tumor population requires to surpass the density $S_{T}^{*}$. As a matter of fact, by imposing $\tilde{S}_{T}^{*}>S_{T}^{*}$ one finds that the following constraints must be simultaneously satisfied

$$
\begin{gathered}
P_{T}^{*}>\frac{\delta_{H} \gamma_{T} \tilde{k}}{\gamma_{H} \epsilon^{2}}, \\
1+\frac{\delta_{T}}{\gamma_{T}}\left(1-\frac{\gamma_{T} \delta_{H} \tilde{k}}{\delta_{T} \gamma_{H} \epsilon}\right)<\psi<\psi_{c} .
\end{gathered}
$$

This situation is shown in Fig. 5, where again the dashed lines account for the set of points of vanishing time derivative of the tumor population while the dashed-dotted 
lines represent the corresponding vanishing time derivative of the normal population. Solid lines describe integral curves. Figure 5a illustrates the effect of the resection when the above constraints are satisfied, whereas the subplot (b) accounts for the extinction of population of tumor cells in the secondary habitat after the resection of the primary tumor.

\subsection{Relation between tumor malignancy and parameter values}

Some of the parameters furnishing our model, such as growth and death rates: $\hat{\Gamma} \equiv$ $\Psi \Gamma_{T}, \hat{\gamma} \equiv \psi \gamma_{T}, \Delta_{T}, \delta_{T}, \Gamma_{H}, \gamma_{H}$, can be reasonably determined upon some available experimental data. Nevertheless, there are five of them: $\Psi, \psi, k, \tilde{k}$ and $\epsilon$ for which the biological information available is still limited.

While $P_{T}^{*}$ and $S_{T}^{*}$ give an idea of how proliferative the tumors are, they can not be taken as a direct measure of their malignancy. Within our model, a more accurate measure of the malignancy of a tumor can be given by the constraints (10) which indeed dictate when the resection of the primary tumor will induce the outgrowth of the local (and probably non observable) metastases. These constraints allow us to establish the region within the space of the 'unknown' parameters where the metastastatic cascade is induced after the resection of the primary tumor. Let us define the following useful quantities

$$
\begin{aligned}
A & =\frac{\delta_{H} \tilde{k}}{\gamma_{H} \epsilon}, \\
B & =\frac{P_{T}^{*} \epsilon}{\hat{\gamma}\left(1-\frac{\delta_{T}}{\hat{\gamma}}\right)}, \\
C & =\sqrt{\frac{\delta_{H} \tilde{k} P_{T}^{*}}{\gamma_{H} \hat{\gamma}\left(1-\frac{\delta_{T}}{\hat{\gamma}}\right)},} \\
\rho_{0} & =\left(\begin{array}{l}
\left.-\frac{C}{1-B}+\sqrt{\frac{C^{2}}{(1-B)^{2}}+\frac{1}{1-B}}\right)^{2}, \\
\rho_{ \pm}
\end{array}\right)^{2} \\
\bar{S} & =\left\{\begin{array}{l}
\frac{C}{B-A} \pm \sqrt{\frac{A}{(B-1)^{2}}-\frac{1}{B-1}} \\
\frac{A}{B}, \quad \text { if } A \leq \frac{B}{1+B}, \\
A>\frac{B}{1+B} .
\end{array}\right.
\end{aligned}
$$

Then, for given values of $P_{T}^{*}, \tilde{k}$ and $\epsilon$, the metastatic outgrowth after the resection of the primary tumor is triggered whenever the malignant fixed point within the secondary habitat, $S_{T}^{*}$, lies in the set 


$$
\begin{cases}B>1+C^{2}, \quad & S_{T}^{*} \in(\bar{S}, \infty) \\ 1<B \leq 1+C^{2}, & \begin{cases}\bar{S} \geq \rho_{+}, & S_{T}^{*} \in(\bar{S}, \infty) \\ \rho_{-} \leq \bar{S}<\rho_{+}, & S_{T}^{*} \in\left(\rho_{+}, \infty\right) \\ \bar{S}<\rho_{-}, & S_{T}^{*} \in\left(\bar{S}, \rho_{-}\right) \cup\left(\rho_{+}, \infty\right)\end{cases} \\ B=1, & \begin{cases}\frac{1}{4 C^{2}}>\bar{S}, & S_{T}^{*} \in\left(\bar{S}, \frac{1}{4 C^{2}}\right) \\ \frac{1}{4 C^{2}} \leq \bar{S}, & \text { No metastatic cascade }\end{cases} \\ B<1, & \begin{cases}\rho_{0}>\bar{S}, & S_{T}^{*} \in\left(\bar{S}, \rho_{0}\right) \\ \rho_{0} \leq \bar{S}, & \text { No metastatic cascade }\end{cases} \end{cases}
$$

Although this classification of the parameter space in terms of the potential malignancy of the system primary-metastatic tumors is absolutely accurate within our model, it should be taken as a guidance for a possible explanation of the outgrowth of local metastases after the resection of (some types of) primary tumors. The rationale would be that those cancers whose parameters verify the constraints are susceptible to develop metastatic outgrowth after the resection of the primary tumor.

Although the model put forward in this section is very simplistic some of the ideas behind it may be useful for a better understanding of the complex phenomena involved in the outgrowth of metastatic tumors after resection of a primary tumor. Its main interest is that it enabled us to furnish a complete analytical study of various possible metastatic scenarios. Our classification of these scenarios was motivated by what is observed in the natural history of most metastatic cancers. In the following section we will present a more sophisticated model incorporating more malignant phenotypes.

\section{Extension of the model to several malignant phenotypes}

\subsection{General model equations}

In the previous section we have presented and analyzed a simple model for cancer metastasis comprising the signalling link between the primary and the secondary tumors and its predictive capability to account for the possible acceleration of metastatic growth after the resection of the primary tumor. However, it is known that in vivo tumors show a vast phenotypic dispersion even in early stages of cancer progression (Hanahan and Weinberg 2011). This wide phenotypic dispersion constitutes the tumor microenvironment. Accordingly, henceforth we shall extend the previous equations to a more realistic situation by incorporating several tumor phenotypes coexisting with normal cells and necrotic tissue, following the same structure as that of the system given by the Eq. (1) albeit with some differences; the more important new ingredient being the presence of a cascade of mutations among malignant phenotypes within the same habitat in a way to be discussed later. As we shall see, the new model still includes the main features found within the previous simplistic system. Although our model has some common aspects with previous works on parallel mutation and 
selection quasispecies theory (Baake and Wagner 2001; Waclaw et al. 2010), the findings in those works are not applicable to the problem of metastatic progression.

The proposed extension of our model consists of the following system of equations. For the primary tumor

$$
\begin{aligned}
\dot{P}_{i}= & \Gamma_{i} P_{i}\left(\Psi-P_{h}-P_{\dagger}-\sum_{l=1}^{M} P_{l}\right)+\sum_{l=1}^{M} \mathcal{M}_{i l} P_{l}-\sum_{l=1}^{M} \mathcal{M}_{l i} P_{i} \\
& -\Delta_{i} P_{i}-k_{i} P_{i}, \\
\dot{P}_{h}= & \Gamma_{h} P_{h}\left(1-P_{h}-P_{\dagger}-\sum_{l=1}^{M} P_{l}\right)-\Delta_{h} P_{h} \sum_{l=1}^{M} \alpha_{l} P_{l}, \\
\dot{P}_{\dagger}= & -\omega_{p} P_{\dagger}+\sum_{l=1}^{M} \Delta_{l} P_{l}+\Delta_{h} P_{h} \sum_{l=1}^{M} \alpha_{l} P_{l},
\end{aligned}
$$

and for the secondary tumor

$$
\begin{aligned}
\dot{S}_{i}= & \gamma_{i} S_{i}\left(\psi-S_{h}-S_{\dagger}-\sum_{l=1}^{M} S_{l}\right) \\
& +\sum_{l=1}^{M} \mathcal{N}_{i l} S_{l}-\sum_{l=1}^{M} \mathcal{N}_{l i} S_{i}-\delta_{i} S_{i}-\sum_{j=1}^{M} \epsilon_{i j} S_{i} P_{j}+\tilde{k}_{i} P_{i}, \\
\dot{S}_{h}= & \gamma_{h} S_{h}\left(1-S_{h}-S_{\dagger}-\sum_{l=1}^{M} S_{l}\right)-\delta_{h} S_{h} \sum_{l=1}^{M} \beta_{l} S_{l}, \\
\dot{S}_{\dagger}= & -\omega_{s} S_{\dagger}+\sum_{l=1}^{M} \delta_{l} S_{l}+\delta_{h} S_{h} \sum_{l=1}^{M} \beta_{l} S_{l} .
\end{aligned}
$$

As pointed out previously, the extended model incorporates $M$ different malignant phenotypes whose population densities are denoted as $P_{i}$, within the primary tumor, and $S_{i}$ for the secondary habitat with $i=1, \ldots, M$. These malignant cells coexist with normal differentiated cells and necrotic tissue, denoted as $P_{h}\left(S_{h}\right)$ and $P_{\dagger}\left(S_{\dagger}\right)$, respectively. In addition, $\Gamma_{i}, \Gamma_{h}, \gamma_{i}$ and $\gamma_{h}$ denote the different proliferation rates while $\Delta_{i}, \Delta_{h}, \delta_{i}$ and $\delta_{h}$ are the death rates. The constants $\omega_{p}$ and $\omega_{s}$ account for the inverse of the characteristic times that the primary and secondary habitats require for complete clearance of the necrotic debris, respectively. The matrices $\mathcal{M}$ and $\mathcal{N}$ represent the transitions among different phenotypes within each of the two habitats. They are defined as $\mathcal{M}_{i j}=P(j \rightarrow i)$, that is: the switching rate from phenotype $j$ to phenotype $i$ within the primary habitat, the matrix $\mathcal{N}$ being analogously defined. ${ }^{1}$

\footnotetext{
1 The probability for phenotype $i$ to not change at all is superfluous as far as the equations are concerned since such diagonal term does not appear in the combination $\sum_{l=1}^{M} \mathcal{M}_{i l} P_{l}-\sum_{l=1}^{M} \mathcal{M}_{l i} P_{i}$. Therefore, without loss of generality we can ignore it.
} 
The transition matrices $\mathcal{M}$ and $\mathcal{N}$ capture the emergence of a new phenotype $i$ from already existing phenotypes $j$, with $i>j$, thus imposing a lineage of phenotypes which can coexist within each tumor for a certain period of time until extinction of a subset of them occurs. The switching processes actually encompass a highly complex dynamical system by itself; the internal cell interactions and the changing environmental conditions playing a key role. As before, the terms $-k_{i} P_{i}$ and $+\tilde{k}_{i} P_{i}$ in Eq. (12a) and (12d), respectively, account for cell migration. The current model naturally assumes the possibility that the values of the migration rates $k_{i}$ and $\tilde{k}_{i}$ do not necessarily increase with the phenotype $i$. The view that metastasis constitutes the final step in a cumulative process of genetic alterations within cells forming a primary tumor lesion has been challenged in recent years (Weinberg 2008; Coghlin and Murray 2010). This has given rise to a new paradigm of metastatic dissemination of cancer cells. For decades, the migration of tumor cells from the primary site to secondary ectopic tissues was regarded as the late-stage event of a sequential process in which genetic and epigenetic changes were acquired by the primary tumor cells over a long period, conferring them a number of capabilities. Namely, loss of cellular adhesion, increased invasiveness, intravasation and survival in the circulatory system, extravasation, survival and proliferation in distant and new tissues from the parent one. An alternative interpretation proposes that the ability to metastasize could be furnished by genetic changes taking place at an early stage during tumorigenesis. Again, in our model this is reflected by the fact that the values of $k_{i}$ and $\tilde{k}_{i}$, though small, could already be significant for low phenotype indexes $i$, thus accounting for early metastatic events.

Notice that Eq. (12d) also includes the generalized term $-\sum_{l} \epsilon_{i l} P_{l} S_{i}$, characterizing the metastasis suppression effect. The 'suppression matrix', $\epsilon_{i j}$, accounts for the fact that the interplay between the metastasis suppressors released by the tumor cells may yield different outcomes depending on the phenotypes involved. Finally, in the death term for the normal cells in Eq. (12e), the constants $\alpha_{i}$ take into account that distinct malignant phenotypes may accelerate the death of normal cells differently; for instance by releasing wastes, such as those that increase the acidification of the tumor microenvironment (Smallbone et al. 2008; Mendoza-Juez et al. 2012).

\subsection{Particular model for a small number of metabolic phenotypes}

Notwithstanding that the model equations (12) allow for an arbitrarily large number of tumor cell phenotypes, it also includes a substantial set of parameters for which biological data are not readily available. Thus, to consider a more complicated scenario than the one described in Sect. 2 while keeping the system size limited, we henceforth particularize the general model (12) to study the evolution of three different malignant phenotypes which will be classified according to their metabolic pathways: oxidative $\left(P_{o}, S_{o}\right)$, glycolytic $\left(P_{g}, S_{g}\right)$ and irreversibly glycolytic $\left(P_{i g}, S_{i g}\right)$ tumor cells.

This is motivated by the well known fact that, during tumorigenesis, the initially well oxygenated population of tumor cells experience an increased number of hypoxic episodes, after reaching a critical size in which the existing vascular network is no longer able to feed the extra cells generated during the neoplastic proliferation (Wilson and Hay 2011). Hypoxia triggers many phenotypical changes but, remarkably, in the 
absence of oxygen cells become predominantly glycolytic to facilitate proliferation and to obtain their energetic resources with a minimal oxygen cost (Gatenby and Gillies 2004; Mendoza-Juez et al. 2012). During angiogenesis, due to the irregular functionality of the tumor-induced neovasculature, cells are subjected to cycles of hypoxia and reoxygenation and, thus, a change of phenotypes occurs until the glycolytic phenotype may eventually end up in an irreversible state, also known as the Warburg phenotype (Warburg et al. 1924; Warburg 1956; Koppenol et al. 2011). Accordingly with that phenomenology, the switching matrices among our three phenotypes will be taken as

$$
\mathcal{M}=\left(\begin{array}{ccc}
0 & M_{\text {og }} & 0 \\
M_{g o} & 0 & 0 \\
0 & M_{i g g} & 0
\end{array}\right), \quad \mathcal{N}=\left(\begin{array}{ccc}
0 & m_{\text {og }} & 0 \\
m_{g o} & 0 & 0 \\
0 & m_{i g g} & 0
\end{array}\right),
$$

thus leading to a set of model equations of the form

$$
\begin{aligned}
& \left(\begin{array}{c}
\dot{P}_{o} \\
\dot{P}_{g} \\
\dot{P}_{i g}
\end{array}\right)=\left(\begin{array}{ccc}
\Pi_{o} & M_{o g} & 0 \\
M_{g o} & \Pi_{g} & 0 \\
0 & M_{i g g} & \Pi_{i g}
\end{array}\right) \cdot\left(\begin{array}{c}
P_{o} \\
P_{g} \\
P_{i g}
\end{array}\right) \\
& \Pi_{l}=\Gamma_{l}\left(\Psi-P_{h}-P_{\dagger}-\sum_{j \in\{o, g, i g\}} P_{j}\right)-\Delta_{l}-k_{l}-\hat{M}_{l}, \\
& l \in\{o, g, i g\}, \hat{M}_{o}=M_{g o}, \hat{M}_{g}=M_{o g}+M_{i g g}, \hat{M}_{i g}=0 \\
& \dot{P}_{h}=\Gamma_{h} P_{h}\left(1-P_{h}-P_{\dagger}-\sum_{j \in\{o, g, i g\}} P_{j}\right)-\Delta_{h} P_{h} \sum_{j \in\{o, g, i g\}} \alpha_{j} P_{j},(14 \\
& \dot{P}_{\dagger}=-\omega_{p} P_{\dagger}+\sum_{j \in\{o, g, i g\}} \Delta_{j} P_{j}+\Delta_{h} P_{h} \sum_{j \in\{o, g, i g\}} \alpha_{j} P_{j}, \\
& \left(\begin{array}{c}
\dot{S}_{o} \\
\dot{S}_{g} \\
\dot{S}_{i g}
\end{array}\right)=\left(\begin{array}{ccc}
\pi_{o} & m_{o g} & 0 \\
m_{g o} & \pi_{g} & 0 \\
0 & m_{i g g} & \pi_{i g}
\end{array}\right) \cdot\left(\begin{array}{c}
S_{o} \\
S_{g} \\
S_{i g}
\end{array}\right)+\left(\begin{array}{c}
\tilde{k}_{o} P_{o} \\
\tilde{k}_{g} P_{g} \\
\tilde{k}_{i g} P_{i g}
\end{array}\right), \\
& \pi_{l}=\gamma_{l}\left(\psi-S_{h}-S_{\dagger}-\sum_{j \in\{o, g, i g\}} S_{j}\right)-\delta_{l}-\hat{m}_{l}-\sum_{j \in\{o, g, i g\}} \epsilon_{l j} S_{l} P_{j}, \\
& l \in\{o, g, i g\}, \hat{m}_{o}=m_{g o}, \hat{m}_{g}=m_{o g}+m_{i g g}, \hat{m}_{i g}=0, \\
& \dot{S}_{h}=\gamma_{h} S_{h}\left(1-S_{h}-S_{\dagger}-\sum_{j \in\{o, g, i g\}} S_{j}\right) \\
& -\delta_{h} S_{h} \sum_{j \in\{o, g, i g\}} \beta_{j} S_{j} \\
& \dot{S}_{\dagger}=-\omega_{s} S_{\dagger}+\sum_{j \in\{o, g, i g\}} \delta_{j} S_{j}+\delta_{h} S_{h} \sum_{j \in\{o, g, i g\}} \beta_{j} S_{j} .
\end{aligned}
$$


The metabolic classification and the structure of the switching matrices are further supported by a series of experiments performed by Sonveaux et al. (2008) using two different tumor cell lines: human colorectal adenocarcinoma, which turns out to preferentially follow the glycolytic pathway and human cervix squamous cell carcinoma which, in contrast, is preferentially oxidative. As the experiments show, the cells metabolic behavior may change depending on the surrounding conditions which, in turn, vary with the availability of resources and the presence of oxygen. The inclusion of the competition for the resources, however, does not substantially alter the solutions admitted by the present model, although it makes the treatment of these much more complicated. Thus, for the sake of simplicity, here we codify this phenotypic switch through the above transition matrices. Finally, for the case under consideration, we choose the suppressor matrix to be

$$
\epsilon_{i j}=\left\{\begin{array}{ll}
\epsilon, & \text { if } i=j \\
r \epsilon, & \text { if } i \neq j
\end{array}, \quad 0 \leq r \leq 1\right.
$$

which may describe phenotypic affinity in the growth suppression effect, corresponding to $r<1$.

The steady malignant configuration within the primary habitat (see Appendix 4) verifies the eigenvalue problem

$$
\left(\begin{array}{ccc}
D_{o}+\Lambda\left(\boldsymbol{P}^{*}\right) & \frac{M_{o g}}{\Gamma_{o}} & 0 \\
\frac{M_{g o}}{\Gamma_{g}} & D_{g}+\Lambda\left(\boldsymbol{P}^{*}\right) & 0 \\
0 & \frac{M_{i g g}}{\Gamma_{i g}} & D_{i g}+\Lambda\left(\boldsymbol{P}^{*}\right)
\end{array}\right) \cdot\left(\begin{array}{c}
P_{o}^{*} \\
P_{g}^{*} \\
P_{i g}^{*}
\end{array}\right)=\left(\begin{array}{l}
0 \\
0 \\
0
\end{array}\right)
$$

with

$$
\begin{aligned}
D_{o} & =-\frac{\Delta_{o}+M_{g o}+k_{o}}{\Gamma_{o}}, \\
D_{g} & =-\frac{\Delta_{g}+M_{o g}+M_{i g g}+k_{g}}{\Gamma_{g}}, \\
D_{i g} & =-\frac{\Delta_{i g}+k_{i g}}{\Gamma_{i g}}, \\
\Lambda\left(\boldsymbol{P}^{*}\right) & =\Psi-1+\frac{\Delta_{h}}{\Gamma_{h}}\left(\alpha_{o} P_{o}^{*}+\alpha_{g} P_{g}^{*}+\alpha_{i g} P_{i g}^{*}\right),
\end{aligned}
$$

and the eigenvalues are given by

$$
D_{i g}, \Lambda_{ \pm}=\frac{1}{2}\left(D_{o}+D_{g} \pm \sqrt{\left(D_{o}-D_{g}\right)^{2}+4 \frac{M_{g o} M_{o g}}{\Gamma_{o} \Gamma_{g}}}\right)
$$


The generic stationary solution for the malignant populations hence reads

$$
\begin{aligned}
P_{g}^{*} & =-\frac{\Gamma_{i g}}{M_{i g g}}\left(D_{i g}-\Lambda\right) P_{i g}^{*}, \\
P_{o}^{*} & =\frac{\Gamma_{g} \Gamma_{i g}}{M_{g o} M_{i g g}}\left(D_{i g}-\Lambda\right)\left(D_{g}-\Lambda\right) P_{i g}^{*}, \\
P_{i g}^{*} & =\frac{1-\Psi-\Lambda}{\frac{\Delta_{h}}{\Gamma_{h}}\left[\alpha_{i g}+\alpha_{g} \hat{P}_{g}+\alpha_{o} \hat{P}_{o}\right]}, \\
\hat{P}_{g} & =-\frac{\Gamma_{i g}}{M_{i g g}}\left(D_{i g}-\Lambda\right), \\
\hat{P}_{o} & =\frac{\Gamma_{g} \Gamma_{i g}}{M_{g o} M_{i g g}}\left(D_{i g}-\Lambda\right)\left(D_{g}-\Lambda\right),
\end{aligned}
$$

with $\Lambda \in\left\{D_{i g}, \Lambda_{ \pm}\right\}$.

For $\Lambda=D_{i g}$ we have an asymptotically 'specialized' malignant population within the primary tumor where only the Warburg phenotype is present, while for $\Lambda=\Lambda_{ \pm}$ an asymptotical coexistence between all metabolic pathways is achieved. In the next subsection, however, we will see that this last solution is not reachable from a non negative initial condition since, in general, it contains negative components. As a matter of fact, $D_{i g}-\Lambda_{ \pm}$is in general positive.

For the secondary malignant populations one finds the equation

$$
\left(\begin{array}{ccc}
d_{o}+\lambda & m_{o g} / \gamma_{o} & 0 \\
m_{g o} / \gamma_{g} & d_{g}+\lambda & 0 \\
0 & m_{i g g} / \gamma_{i g} & d_{i g}+\lambda
\end{array}\right) \cdot\left(\begin{array}{c}
S_{o}^{*} \\
S_{g}^{*} \\
S_{i g}^{*}
\end{array}\right)=-\left(\begin{array}{c}
\tilde{k}_{o} P_{o}^{*} / \gamma_{o} \\
\tilde{k}_{g} P_{g}^{*} / \gamma_{g} \\
\tilde{k}_{i g} P_{i g}^{*} / \gamma_{i g}
\end{array}\right),
$$

with

$$
\begin{aligned}
& d_{o}=-\frac{m_{g o}}{\gamma_{o}}-\frac{\delta_{o}}{\gamma_{o}}-\frac{\epsilon}{\gamma_{o}}\left[r\left(P_{g}^{*}+P_{i g}^{*}\right)+P_{o}^{*}\right], \\
& d_{g}=-\frac{m_{o g}}{\gamma_{g}}-\frac{m_{i g g}}{\gamma_{g}}-\frac{\delta_{g}}{\gamma_{g}}-\frac{\epsilon}{\gamma_{g}}\left[r\left(P_{o}^{*}+P_{i g}^{*}\right)+P_{g}^{*}\right], \\
& d_{i g}=-\frac{\delta_{i g}}{\gamma_{i g}}-\frac{\epsilon}{\gamma_{i g}}\left[r\left(P_{o}^{*}+P_{g}^{*}\right)+P_{i g}^{*}\right],
\end{aligned}
$$

and $\lambda$ given by the restriction

$$
\lambda=\psi-1+\frac{\delta_{h}}{\gamma_{h}}\left(\beta_{o} S_{o}^{*}+\beta_{g} S_{g}^{*}+\beta_{i g} S_{i g}^{*}\right)
$$

Similarly to the primary habitat, the eigenvalues are given by $d_{i g}$ and

$$
\lambda_{ \pm}=\frac{1}{2}\left(d_{o}+d_{g} \pm \sqrt{\left(d_{o}-d_{g}\right)^{2}+4 \frac{m_{g o} m_{o g}}{\gamma_{o} \gamma_{g}}}\right) .
$$


At this point we find two non equivalent solutions. If $\lambda \notin\left\{-d_{i g},-\lambda_{ \pm}\right\}$we obtain a 'deterministic solution' where the asymptotic populations within the secondary habitat are

$$
\begin{aligned}
& S_{o}^{*}=-\frac{\left(d_{g}+\lambda\right) \tilde{k}_{o} P_{o}^{*}-\frac{m_{o g}}{\gamma_{o}} \tilde{k}_{g} P_{g}^{*}}{\left(d_{o}+\lambda\right)\left(d_{g}+\lambda\right)-\frac{m_{o g} m_{g o}}{\gamma_{g} \gamma_{o}}}, \\
& S_{g}^{*}=-\frac{\left(d_{o}+\lambda\right) \tilde{k}_{g} P_{g}^{*}-\frac{m_{g o}}{\gamma_{g}} \tilde{k}_{o} P_{o}^{*}}{\left(d_{o}+\lambda\right)\left(d_{g}+\lambda\right)-\frac{m_{o g} m_{g o}}{\gamma_{g} \gamma_{o}}}, \\
& S_{i g}^{*}=-\frac{\tilde{k}_{i g} P_{i g}^{*}+\frac{m_{i g g}}{\gamma_{i g}} S_{g}^{*}}{d_{i g}+\lambda}, \\
& S_{h}^{*}=\frac{1-\sum_{l \in\{o, g, i g\}}\left(1+\frac{\delta_{l}}{\omega_{s}}-\frac{\delta_{h}}{\gamma_{h}} \beta_{l}\right) S_{l}^{*}}{1+\frac{\delta_{h}}{\omega_{s}} \sum_{l \in\{o, g, i g\}} \beta_{l} S_{l}^{*}} .
\end{aligned}
$$

The term deterministic reflects the fact that the matrix in Eq. (19a) has a non vanishing determinant. As a matter of fact, this solution follows the structure of the primary solution in the sense that coexistence arises only if it already appears within the primary habitat.

There is still a 'singular solution' for the secondary habitat if the following (geometric) conditions

$$
\begin{gathered}
\left(\begin{array}{c}
\frac{\tilde{k}_{o}}{\gamma_{o}} P_{o}^{*} \\
\frac{\tilde{k}_{g}}{\gamma_{g}} P_{g}^{*} \\
\frac{\tilde{k}_{i g}}{\gamma_{i g}} P_{i g}^{*}
\end{array}\right)^{T} \cdot\left(\begin{array}{c}
\frac{m_{i g g} m_{g o}}{\gamma_{i g} \gamma_{g}} \\
\frac{m_{i g g}}{\gamma_{i g}}\left(d_{o}+\lambda\right) \\
\left(d_{o}+\lambda\right)\left(d_{g}+\lambda\right)-\frac{m_{o g} m_{g o}}{\gamma_{o} \gamma_{g}}
\end{array}\right)=0, \\
\left(d_{i g}+\lambda\right)\left[\left(d_{o}+\lambda\right)\left(d_{g}+\lambda\right)-\frac{m_{o g} m_{g o}}{\gamma_{o} \gamma_{g}}\right]=0,
\end{gathered}
$$

are met. In particular, for $P_{o}^{*}=P_{g}^{*}=0$ and $\left(d_{o}+\lambda\right)\left(d_{g}+\lambda\right)-m_{o g} m_{g o} /\left(\gamma_{o} \gamma_{g}\right)=0$ the singular solution is given by the equations

$$
\begin{aligned}
S_{o}^{*} & =-\frac{\gamma_{g}}{m_{g o}}\left(d_{g}+\lambda\right) S_{g}^{*}, \\
S_{i g}^{*} & =-\frac{1}{d_{i g}+\lambda}\left(\frac{\tilde{k}_{i g}}{\gamma_{i g}} P_{i g}^{*}+\frac{m_{i g g}}{\gamma_{i g}} S_{g}^{*}\right), \\
S_{g}^{*} & =\frac{\psi-\lambda+\left(1+\frac{\delta_{i g}}{\omega_{s}}\right) \frac{\tilde{k}_{i g} P_{i g}^{*}}{\gamma_{i g}\left(d_{i g}+\lambda\right)}}{1+\frac{\delta_{g}}{\omega_{s}}-\left(1+\frac{\delta_{o}}{\omega_{s}}\right) \frac{\gamma_{g}\left(d_{g}+\lambda\right)}{m_{g o}}-\left(1+\frac{\delta_{i g}}{\omega_{s}}\right) \frac{m_{i g g}}{\gamma_{i g}\left(d_{i g}+\lambda\right)}}, \\
S_{h}^{*} & =0 .
\end{aligned}
$$


It can be shown that, since $P_{i g}^{*}>0$, the singular steady solution corresponding to $d_{i g}+\lambda=0$ from the geometric constraint (21b) necessarily has negative components and thus it cannot be reached by any non negative initial value of the population densities (see Appendix 4).

The above phenotypically diverse asymptotic solution is somehow a surprising equilibrium since, even when from the primary tumor only the Warburg phenotype (which is a steady phenotype in both habitats) is asymptotically migrating, we may still find a phenotypically sparse population within the secondary habitat.

\subsection{Parameter estimation and numerical studies of the solutions}

In this section we will present a set of numerical simulations of the solutions allowed by the model given by Eq. (14). As we shall see, the basic features arising within the simple model having a single tumor phenotype in Sect. 2 are also found within this extended model. In particular, we still obtain the existence of a threshold for the secondary carrying capacity dividing the extinction of the population of normal cells from the coexistence equilibrium and, as far as the resection of the primary tumor is concerned, again we find a window for the metastatic cascade to occur within the secondary habitat. To estimate the proliferation/death constants of malignant cells we use the fact that, for population densities close to zero, the evolution equations adopt the generic simple form $\dot{n} / n=\Gamma-\Delta$ from which the time of doubling population verifies $\Gamma-\Delta=\frac{\ln 2}{T_{2}}$. Moreover, the corresponding necrotic population increases according to $n_{\dagger}(t) / n(t)=\frac{\Delta T_{2}}{\ln 2}\left(1-\exp \left(-t \ln 2 / T_{2}\right)\right)$.

It is well known that glycolytic phenotypes are typically more proliferative (Van der Heiden et al. 2010) than oxidative ones. We will thus take a doubling time of 2 days for oxidative cells while for glycolytic phenotypes we will consider a doubling time of 1 day. In addition, the cell death fraction can be estimated to be around $7 \%$ common to both oxidative and glycolytic phenotypes. For the normal cells the doubling times have an enormous variance from about 1 day to years depending on the specific type of tissue. For epithelial tissues we will take a doubling time of around 4 days. Finally, we will assume that the time needed by both habitats to absorb half of the necrotic population is around 3 days. That is: $\omega_{p}=\omega_{s} \simeq 0.23$ day $^{-1}$. Table 2 lists the parameter values chosen for the simulations presented in this section for the primary and secondary habitats and some of the derived quantities.

From the values of Table 2 we get $D_{i g}-\Lambda_{+} \simeq 0.02, D_{i g}-\Lambda_{-} \simeq 70.5$ and hence for the primary habitat only the Warburg-like phenotype is asymptotically selected. As a consequence, the secondary malignant deterministic steady solutions given in Eq. (20) reduce to

$$
S_{o}^{*}=S_{g}^{*}=0, \quad S_{i g}^{*}=-\frac{\tilde{k}_{i g} P_{i g}^{*}}{\theta},
$$

with $\theta$ verifying the constraint $\theta^{2}-\left(\psi-1+d_{i g}\right) \theta+\frac{\delta_{h} \tilde{k}_{i g}}{\gamma_{h} \gamma_{i g}} \beta_{i g} P_{i g}^{*}=0$, which has real (and negative) solutions only for 
Table 2 Parameter values and derived quantities for the primary and secondary tumor used in the simulations of Sect. 3.3

\begin{tabular}{|c|c|c|c|c|c|}
\hline \multicolumn{2}{|l|}{ Primary tumor } & \multicolumn{2}{|c|}{ Secondary tumor } & \multirow[t]{2}{*}{ Units } & \multirow[t]{2}{*}{ Remark } \\
\hline Quantity & Value & Quantity & Value & & \\
\hline$\Gamma_{o} \psi$ & 0.4 & $\gamma_{o} \psi$ & 0.4 & day $^{-1}$ & \\
\hline$\Gamma_{g} \psi=\Gamma_{i g} \psi$ & 0.8 & $\gamma_{g} \psi=\gamma_{i g} \psi$ & 1 & day $^{-1}$ & \\
\hline$\Gamma_{h}$ & 0.2 & $\gamma_{h}$ & 0.2 & day $^{-1}$ & See Sonveaux et al. ( 2008), \\
\hline$\Delta_{o}$ & 0.04 & $\delta_{o}$ & 0.05 & day $^{-1}$ & Mendoza-Juez et al. (2012) \\
\hline$\Delta_{g}=\Delta_{i g}$ & 0.06 & $\delta_{g}=\delta_{i g}$ & 0.1 & day $^{-1}$ & and notes on text \\
\hline$\Delta_{h}$ & 1 & $\delta_{h}$ & 1 & day $^{-1}$ & \\
\hline$\omega_{p}$ & 0.2 & $\omega_{s}$ & 0.2 & day $^{-1}$ & \\
\hline$k_{o}$ & 0 & $\tilde{k}_{o}$ & 0 & day $^{-1}$ & \\
\hline$k_{g}=k_{i g}$ & $10^{-3}$ & $\tilde{k}_{g}=\tilde{k}_{i g}$ & $10^{-4}$ & day $^{-1}$ & \\
\hline$M_{o g}$ & 5 & $m_{o g}$ & 5 & day $^{-1}$ & \\
\hline$M_{g o}$ & 20 & $m_{g o}$ & 20 & day $^{-1}$ & Estimated \\
\hline$M_{i g g}$ & 0.1 & $m_{i g g}$ & 0.1 & day $^{-1}$ & \\
\hline$\alpha_{o}$ & 0.1 & $\beta_{o}$ & 0.1 & & \\
\hline$\alpha_{g}=\alpha_{i g}$ & 1 & $\beta_{g}=\beta_{i g}$ & 1 & & \\
\hline$\Psi$ & 1.2 & $\epsilon$ & 0.056 & day $^{-1}$ & \\
\hline - & - & $r$ & 0.5 & & \\
\hline
\end{tabular}

$$
\epsilon / \gamma_{i g} \geq \frac{\psi-1+2 \sqrt{\delta_{h} \beta_{i g} \tilde{k}_{i g} P_{i g}^{*} /\left(\gamma_{h} \gamma_{i g}\right)}-\frac{\delta_{i g}}{\gamma_{i g}}}{P_{i g}^{*}} \simeq 0.05 .
$$

On the other hand, for the singular solution given in Eq. (22) to be non negative we need $d_{g}+\lambda \leq 0$ and $d_{i g}+\lambda<0$. The first constraint is guaranteed by one of the solutions to the restriction $\left(d_{o}+\lambda\right)\left(d_{g}+\lambda\right)-m_{o g} m_{g o} /\left(\gamma_{o} \gamma_{g}\right)=0$. The second constraint, however, requires

$$
\begin{aligned}
\epsilon / \gamma_{i g} & >\frac{B-\sqrt{B^{2}-4 A C}}{2 A P_{i g}^{*}} \simeq 0.032, \\
A & =(1-r)\left(1-r \frac{\gamma_{g}}{\gamma_{o}}\right), \\
B & =\frac{m_{o g}+m_{i g g}}{\gamma_{g}}\left(1-r \frac{\gamma_{g}}{\gamma_{o}}\right)+(1-r)\left(\frac{\delta_{o}}{\gamma_{o}}-\frac{\delta_{g}}{\gamma_{g}}+\frac{m_{g o}}{\gamma_{o}}\right), \\
C & =\frac{m_{g o} m_{i g g}}{\gamma_{g} \gamma_{o}}+\frac{m_{o g}+m_{i g g}}{\gamma_{g}}\left(\frac{\delta_{o}}{\gamma_{o}}-\frac{\delta_{g}}{\gamma_{g}}\right) .
\end{aligned}
$$

In the following subsection we will numerically show the existence of these thresholds. 
3.4 Threshold for the carrying capacity $\psi$ in the secondary habitat

In this subsection we present numerical simulations confirming the existence of a threshold for the carrying capacity, $\psi$, in the secondary habitat already found in the single-species tumor model. Our results are summarized in Figs. 6 and 7. In all of the plots, the solid curves represent the normal cell population while the tumor population is represented by dashed lines with the following particular assignment: from thinnest to thickest, the dashed lines represent oxidative, glycolytic and Warburg phenotypes, respectively.

Figure 6a shows numerical solutions for the primary habitat where the initial condition is 70 of normal cells, 20 of oxidative tumor cells and 10 of glycolytic cancer cells. This choice corresponds to an early stage of the tumor development where most of the (still not dominant) tumor population are oxidative. In addition, we have taken $\Psi=1.2$. Figures $6 \mathrm{~b}$, c display the corresponding solution for the secondary tumor where the initial value is, instead, taken to be $S_{o}(0)=S_{g}(0)=S_{i g}(0)=S_{\dagger}(0)=0$ and $S_{h}(0)=1$. As the graphics thereby show, we still find a critical value for the secondary carrying capacity, $\psi$, which, for the case under consideration, takes the value $\psi_{c} \simeq 1.104$. Notice that below such threshold the cancer cells proliferate to an equilibrium with normal cells in which the latter population is much larger [see Fig. 6c]. Whereas above the threshold the proliferation of cancer cells induce the extinction of the normal population after some time (Fig. 6b) and the malignant population asymptotically approaches a coexistence between all three metabolic phenotypes, corresponding to the singular steady solution given in Eq. (22). Figure 7, on the other hand, shows the (stable) steady solutions within the secondary habitat as a function of the suppressor rate, $\epsilon$. Figure 7 a displays the transition between tumor extinction and the extinction of normal cells taking place around $\epsilon / \gamma_{i g} \sim 0.032$ (constraint (24)) where the malignant steady population turns out to be the metabolic coexisting equilibrium given in Eq. (22). Figure $7 \mathrm{~b}$ depicts the transition between tumor extinction and the malignant-normal coexistence at a value of $\epsilon / \gamma_{i g} \sim 0.05$ (cf. Eq. (23)). Notice that the malignant component within the coexistence equilibrium corresponds to the deterministic steady solution given in Eq. (20). Moreover, in analogy with the coexistence equilibrium found with the single tumor phenotype model in Sect. 2 (cf. Fig. 2), only the upper branch solution (the one with lowest malignant component) turns out to be a stable solution.

\subsection{Effect of the primary resection on local metastases}

The effect of the resection of the primary tumor is shown in Fig. 8. Figure 8a shows the solution for the secondary habitat for $\psi=1.1<\psi_{c}$, corresponding to the solution for the primary habitat shown in Fig. 6a, where a metastatic cascade takes place around 130 days after the resection of the primary tumor. In this case the metastatic evolution selects the Warburg phenotype to survive, as it corresponds to the steady state of the primary habitat, since after the resection the interaction with the primary tumor is obviously switched off. Nevertheless, if we take $\psi=1.05$ instead, the effect of the resection of the primary tumor on the local metastasis is the extinction of the 

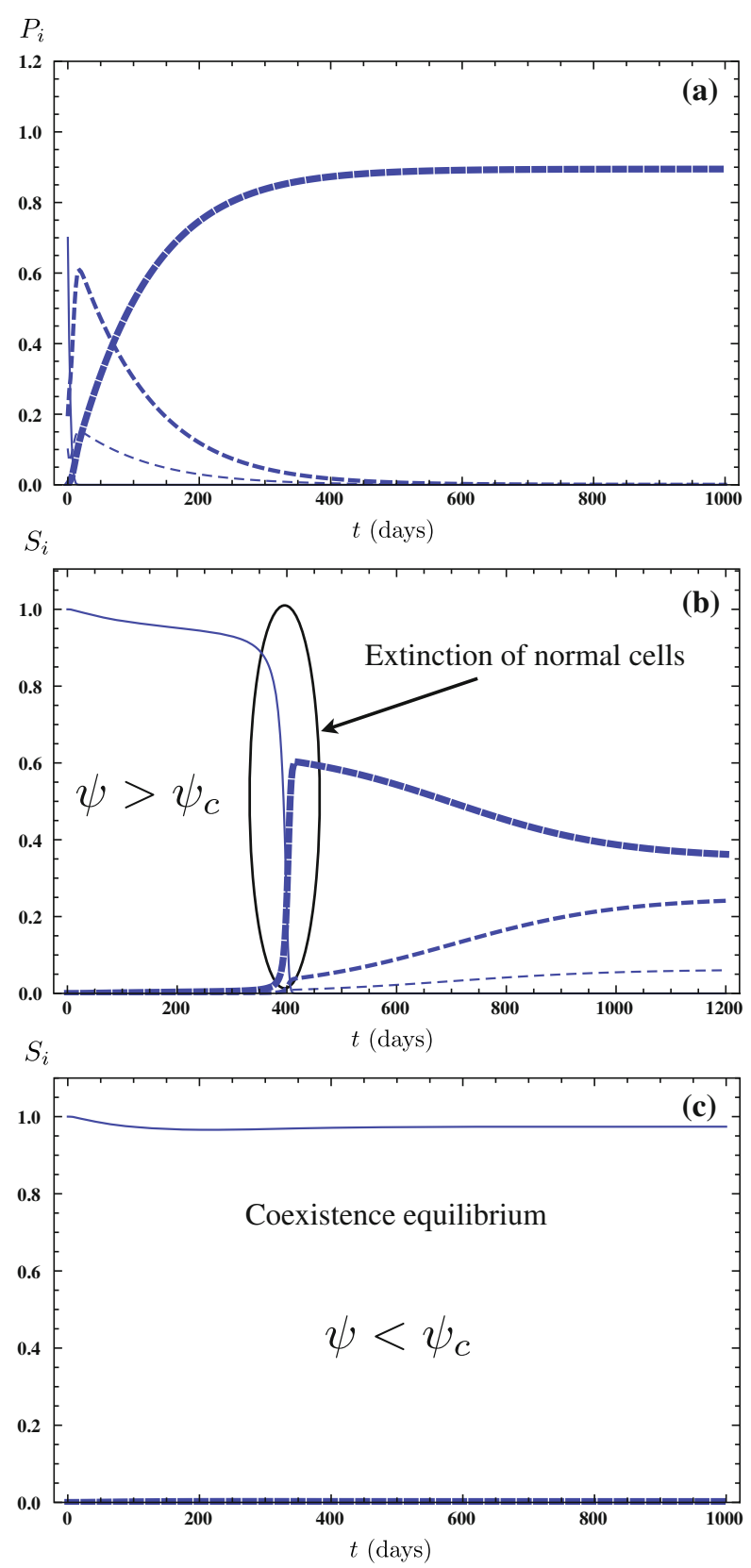

Fig. 6 a Solution for the primary habitat for $\psi=1.2$ and initial values $P_{g}(0)=0.1, P_{o}(0)=0.2$, $P_{i g}(0)=0, P_{h}(0)=0.7$ and $P_{\dagger}(0)=0$. The dashed lines represent, from thinnest to thickest, oxidative tumor cells, glycolytic malignant cells and Warburg phenotype, respectively. The solid line describes the normal cells. b Corresponding solution for the secondary habitat with a carrying capacity value above the threshold $(\psi=1.105)$. c Corresponding solution for the secondary habitat with a carrying capacity value below the threshold $(\psi=1.1)$. In both cases the initial values are: $S_{o}(0)=S_{g}(0)=S_{i g}(0)=S_{\dagger}(0)=0$ and $S_{h}(0)=1$ and the line-type distribution is the same as that in the subplot (a) 


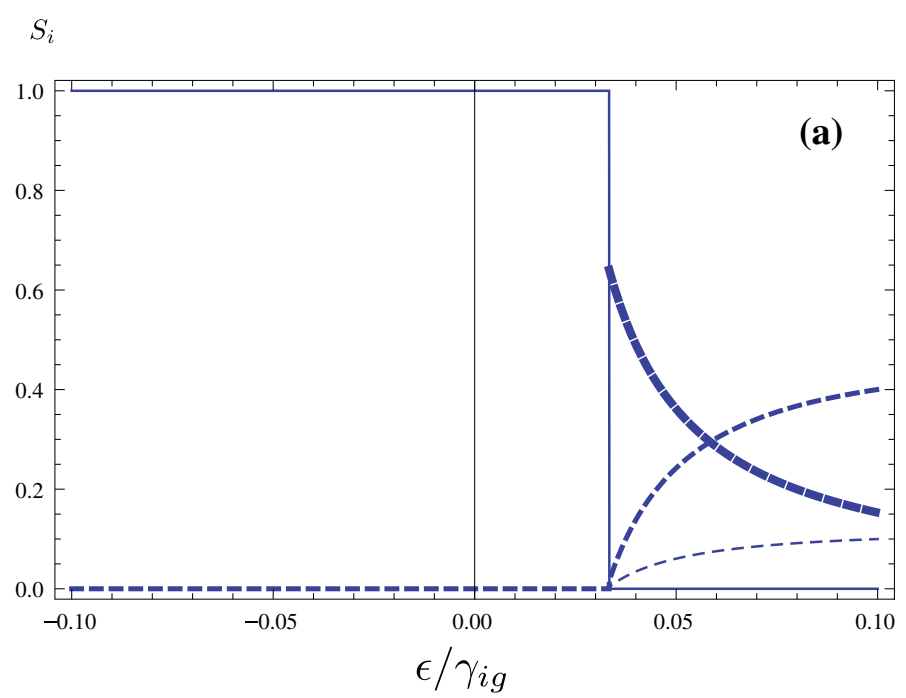

$S_{i}$

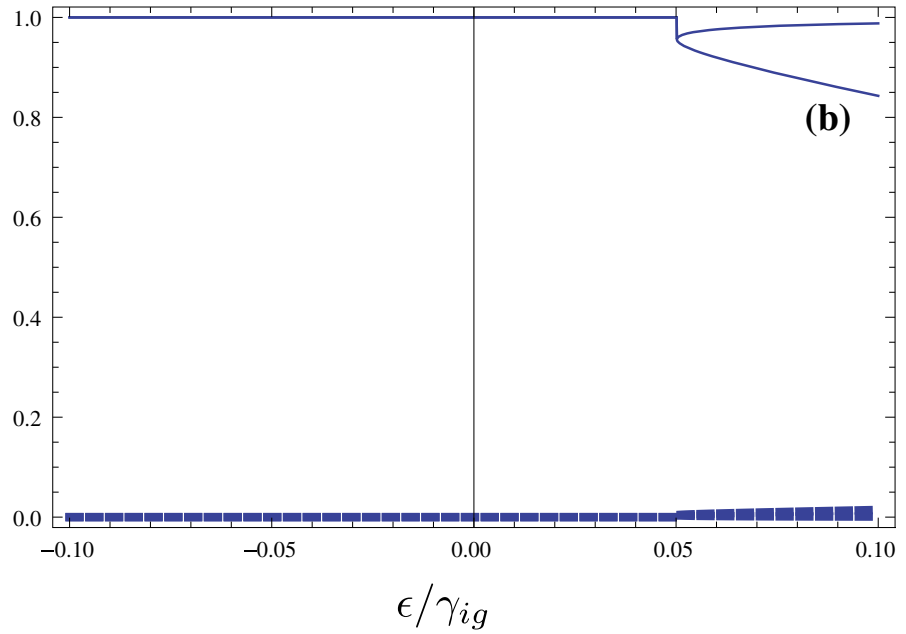

Fig. 7 Diagram of stationary solutions for the secondary habitat as a function of $\epsilon$. a Transition to the extinction of normal cells with a coexistence among all three malignant phenotypes whenever $\epsilon / \gamma_{i g} \gtrsim 0.032$. This stationary solution turns out to be stable. b Transition to the coexistence equilibrium between the Warburg-like phenotype and the normal cells for $\epsilon / \gamma_{i g} \gtrsim 0.05$. Analogously to the simplified model in Sect. 2, only the upper branch is a stable solution. The coexistence with lower normal cell density (lower branch) is an unstable solution. In both graphics $\psi=1.105$

malignant cells (Fig. 8b), confirming that the metastatic outgrowth after the resection of the primary tumor occurs under certain conditions as found with the single tumor model developed in Sect. 2. 

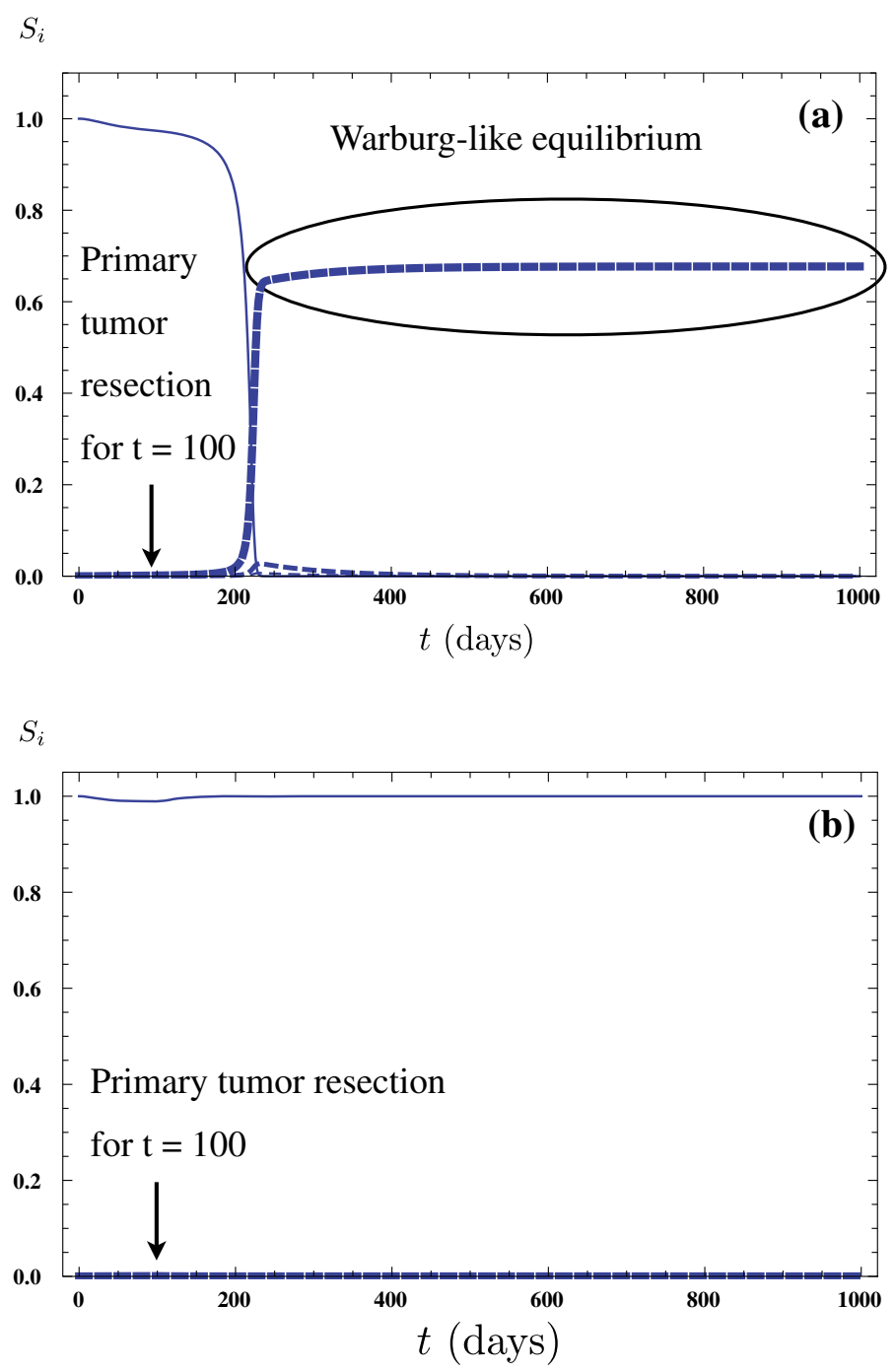

Fig. 8 Solutions for the secondary habitat with the resection of the primary tumor taking place 100 days after the initiation. a Solutions for $\psi=1.1<\psi_{c}$. In this case a metastatic cascade is triggered around 130 days after the resection of the primary tumor. b Solutions for a carrying capacity value $\psi=1.05$. In this case, the resection of the primary tumor induces the extinction of the cancer cells within the metastatic tissue. In both cases the resection of the primary tumor takes place 100 days after the emergence of the malignant cells within the primary habitat

\section{Conclusions and perspectives}

In this paper we have addressed the issue of communication between the primary and metastatic tumors from an evolutionary perspective where the phenomenon of tumor cells dissemination is considered as a migratory event from the primary hab- 
itat to the metastatic site. Furthermore, the growth of the metastasis is assumed to be down-regulated by the interplay between metastasis suppressors secreted by both tumors (Cook et al. 2011). The main results of the $2+2$ model are the following: In the primary habitat only the cancer or the normal cells are the stable solutions, but not both. In contrast, in the secondary habitat, the solutions can exhibit a different stability; there can be a coexistence depending on whether the value of the carrying capacity $\psi$ is smaller or larger than a critical value $\psi_{c}$. The malignant component in the coexisting equilibrium is much smaller than the normal one and, therefore, this coexistence can be interpreted as a dormant metastatic tumor. The effect of the resection of the primary tumor has been considered in the framework of this model and we have found that: if $\psi>\psi_{c}$ there is no impact on the secondary tumor in the sense that the normal population cannot be recovered. If, otherwise, $\psi<\psi_{c}$ there is a window of values for $\psi$, given by Eq. (10), in which the resection triggers the full metastatic invasion of the secondary habitat while, out of the window, the extinction of the secondary tumor occurs. This is in accordance with experimental observations in clinical studies (Ebos et al. 2009; Peeters et al. 2006, 2008, 2004, 2005). In those works, it was observed that, in certain tumor lines, the surgical resection or even chemotherapeutic treatment of the primary tumor was followed, in some cases, by an explosive outgrowth of the local metastases. Our work suggests the existence of distinct regimes where this phenomenon takes place. It is remarkable that even with the inclusion of a term restraining the growth of the secondary tumor whenever the primary tumor is present, the resection of the latter only induces the metastatic explosion under limited circumstances. We want to stress that our present model has not been directly applied to those situations because further biological data are required. Nevertheless, the model is appealing as it provides well defined conditions for such regimes to take place depending on the various parameters (most of them can be reasonably estimated).

Our model has also been extended to several tumor phenotypes while still keeping the same basic features. The main results of the extended model can be summarized as follows: Within the primary habitat the evolution of the populations tends to a steady solution that still presents the extinction of the normal population and where the irreversible glycolytic phenotype (Warburg phenotype) is asymptotically selected among the malignant population (Fig. 6a).

In the secondary habitat, however, the situation is much richer: our numerical simulations, within the explored parameter space, do show the existence of a threshold value for the secondary carrying capacity, $\psi_{c}$, such that below this value the system approaches a coexistence equilibrium between normal and tumor cells in which the malignant population remains in a dormant state (see Fig. 6c). If $\psi>\psi_{c}$ the normal population becomes extinct and the secondary malignant population approaches an equilibrium between all phenotypes. This occurs even when the only phenotype asymptotically migrating from the primary habitat is the Warburg phenotype (see Fig. 6b). The effect of the resection of the primary tumor is also considered within the extended model and we again find a dependence on $\psi$ : depending on its value the resection of the primary tumor may induce the full metastatic invasion of the secondary habitat (Fig. 8a) or, else, the extinction of the secondary tumor (Fig. 8b). In the 
first case, the resection induces the selection of the Warburg phenotype to fully invade the secondary habitat which is a remarkable result.

Our model reflects the phenotypic heterogeneity observed in tumors. It indicates that among the huge variety of cancer cells that may coexist at a given time in a primary tumor, only a selected subpopulation of these may be the relevant one in the outcome of distant metastases. For instance, it has been recently shown that a small population of cancer stem cells is critical for metastatic colonization and that stromal niche signals are crucial to this expansion process (Malanchi et al. 2012).

The understanding of the dependence of the metastatic outgrowth on the different parameters may assist in identifying appropriate therapeutic targets and help in understanding why, beyond other reasons such as physical proximity or 'chemical affinity', some tumors choose to metastasis to specific distant organs and not others. Our multiphenotypic model provides an underlying structure for this behavior. While the main applications have been to present and develop a complete analysis of a family of reduced cases of the general framework, in future works we intend to study specific examples of tumors using data from real metastatic patterns when the primary site is an adenocarcinoma (Hess et al. 2006; DiSibio and French 2008). In that sense, our model could be straightforwardly extended to account for tumor cells migration from a single primary tumor to several secondary sites by simply considering a matrix of migration rates, say: $k_{i}^{j}$, giving rise to a network of metastases (Chen et al. 2009).

As it has been already emphasized, tumors show a huge phenotypic dispersion. In that sense, rather than incorporating a large amount of phenotypes it is more interesting to check whether the present model can be extended to a continuous phenotypic landscape, where the phenotype label is no longer a discrete variable. It is even more interesting to consider higher dimensional phenotypic landscapes, where the different phenotypes may be labeled according to several properties (variables). For that matter we propose, as a first approach, the following landscape extension

$$
\begin{aligned}
\partial_{t} P(t, z)= & \frac{1}{2}(p+q) D \partial_{z}^{2} P(t, z)+v \partial_{z}[(p-q) P(t, z)] \\
& +\Gamma(z) P(t, z)\left[\Psi-P_{h}-P_{\dagger}-\int_{z_{\min }}^{z_{\max }} \mathrm{d} \zeta P(t, \zeta)\right] \\
& -\left(\Delta(z)+\frac{1}{2} D \partial_{z}^{2}(p+q)\right) P(t, z)-k(z) P(t, z), \\
\dot{P}_{h}= & \Gamma_{h} P_{h}\left[1-P_{h}-P_{\dagger}-\int_{z_{\min }}^{z_{\max }} \mathrm{d} \zeta P(t, \zeta)\right] \\
& -\Delta_{h} P_{h} \int_{z_{\min }}^{z_{\max }} \mathrm{d} \zeta \alpha(\zeta) P(t, \zeta),
\end{aligned}
$$




$$
\begin{aligned}
\dot{P}_{\dagger}= & -\omega_{p} P_{\dagger}+\int_{z_{\min }}^{z_{\max }} \mathrm{d} \zeta \Delta(z) P(t, \zeta) \\
& +\Delta_{h} P_{h} \int_{z_{\min }}^{z_{\max }} \mathrm{d} \zeta \alpha(\zeta) P(t, \zeta),
\end{aligned}
$$

and similar formulae for the secondary habitat. The parameters $v=\lim _{\delta t \rightarrow 0} \delta z / \delta t$ and $D=\lim _{\delta t \rightarrow 0} \delta z^{2} / \delta t$ play the role of advection velocity and diffusion coefficient, respectively. The quantity $p$ measures the probability density for a cancer cell to mutate with increasing malignancy (from phenotype $z$ to phenotype $z+\mathrm{d} z$ ) while $q$ measures the probability density mutation towards decreasing malignancy. The suitability and predictive power of the present extension as well as the investigation of possible alternative proposals is left for future works.

Acknowledgments The authors would like to thank Juan Belmonte-Beitia, Alicia Martínez-González, Berta Mendoza-Juez (Universidad de Castilla-La Mancha), Olivier Feron (Universite Catolique de Lovaine) and Luis Pérez-Romasanta (Hospital General de Ciudad Real) for useful discussions and comments. This work has been partially supported by grants MTM2009-13832 (Ministerio de Ciencia e Innovación, Spain) and PEII11-0178-4092 (Junta de Comunidades de Castilla-La Mancha, Spain).

\section{Appendix A: Fixed points and positivity of the solutions}

Consider again the evolution equations for the extended model (12)

$$
\begin{aligned}
\dot{P}_{i}= & \Gamma_{i} P_{i}\left(\Psi-P_{h}-P_{\dagger}-\sum_{l=1}^{M} P_{l}\right) \\
& +\sum_{l=1}^{M} \mathcal{M}_{i l} P_{l}-\sum_{l=1}^{M} \mathcal{M}_{l i} P_{i}-\Delta_{i} P_{i}-k_{i} P_{i}, \\
\dot{P}_{h}= & \Gamma_{h} P_{h}\left(1-P_{h}-P_{\dagger}-\sum_{l=1}^{M} P_{l}\right)-\Delta_{h} P_{h} \sum_{l=1}^{M} \alpha_{l} P_{l}, \\
\dot{P}_{\dagger}= & -\omega_{p} P_{\dagger}+\sum_{l=1}^{M} \Delta_{l} P_{l}+\Delta_{h} P_{h} \sum_{l=1}^{M} \alpha_{l} P_{l}, \\
\dot{S}_{i}= & \gamma_{i} S_{i}\left(\psi-S_{h}-S_{\dagger}-\sum_{l=1}^{M} S_{l}\right) \\
& +\sum_{l=1}^{M} \mathcal{N}_{i l} S_{l}-\sum_{l=1}^{M} \mathcal{N}_{l i} S_{i}-\delta_{i} S_{i}-\sum_{l=1}^{M} \epsilon_{i l} S_{i} P_{l}+\tilde{k}_{i} P_{i}, \\
\dot{S}_{h}= & \gamma_{h} S_{h}\left(1-S_{h}-S_{\dagger}-\sum_{l=1}^{M} S_{l}\right)-\delta_{h} S_{h} \sum_{l=1}^{M} \beta_{l} S_{l},
\end{aligned}
$$




$$
\dot{S}_{\dagger}=-\omega_{S} S_{\dagger}+\sum_{l=1}^{M} \delta_{l} S_{l}+\delta_{h} S_{h} \sum_{l=1}^{M} \beta_{l} S_{l},
$$

For the primary tumor, from the vanishing of Eq. (26b) we deduce the form of the asymptotic population densities to be

$$
\begin{gathered}
\sum_{j=1}^{M} \frac{1}{\Gamma_{i}} \mathcal{M}_{i j} P_{j}-\frac{1}{\Gamma_{i}}\left(\sum_{l=1}^{M} \mathcal{M}_{l i}+\Delta_{i}+k_{i}\right) P_{i}=-\Lambda(\boldsymbol{P}) P_{i}, \\
{\left[1+\frac{\Delta_{h}}{\omega_{p}} \sum_{l=1}^{M} \alpha_{l} P_{l}\right] P_{h}=1-\frac{\Delta_{h}}{\Gamma_{h}} \sum_{l=1}^{M} \alpha_{l} P_{l}-\sum_{l=1}^{M}\left(1+\frac{\Delta_{l}}{\omega_{p}}\right) P_{l},} \\
\omega_{p} P_{\dagger}=\sum_{l=1}^{M} \Delta_{l} P_{l}+\Delta_{h} P_{h} \sum_{l=1}^{M} \alpha_{l} P_{l},
\end{gathered}
$$

where the eigenvalue reads

$$
\Lambda(\boldsymbol{P})=\Psi-1+\frac{\Delta_{h}}{\Gamma_{h}} \sum_{i=1}^{M} \alpha_{i} P_{i} .
$$

For the secondary tumor we have similar expressions

$$
\begin{gathered}
\sum_{j=1}^{M} \frac{1}{\gamma_{i}} \mathcal{N}_{i j} S_{j}-\frac{1}{\gamma_{i}}\left(\sum_{l=1}^{M} \mathcal{N}_{l i}+\delta_{i}+\sum_{l=1}^{M} \epsilon_{i l} P_{l}\right) S_{i}+\lambda(\boldsymbol{S}) S_{i}=-\frac{\tilde{k}_{i}}{\gamma_{i}} P_{i}, \\
{\left[1+\frac{\delta_{h}}{\omega_{s}} \sum_{l=1}^{M} \beta_{l} S_{l}\right] S_{h}=1-\sum_{l=1}^{M}\left(1+\frac{\delta_{l}}{\omega_{s}}\right) S_{l}-\frac{\delta_{h}}{\gamma_{h}} \sum_{l=1}^{M} \beta_{l} S_{l},} \\
\omega_{s} S_{\dagger}=\sum_{l=1}^{M} \delta_{l} S_{l}+\delta_{h} S_{h} \sum_{l=1}^{M} \beta_{l} S_{l},
\end{gathered}
$$

where the function $\lambda(\boldsymbol{S})$ is given by

$$
\lambda(\boldsymbol{S})=\psi-1+\frac{\delta_{h}}{\gamma_{h}} \sum_{i=1}^{M} \beta_{i} S_{i} .
$$

In a stationary situation the system will reach one of the possible steady-state solutions found above. However, some of these have negative components, as we we have shown in Sect. 3.3. Nonetheless, we will prove that the above system of ODEs under consideration leaves the positive quadrant invariant, that is: any non negative initial value will reach a non negative steady solution. 
Let us define $\mathbb{R}_{+}^{n}$ as:

$$
\mathbb{R}_{+}^{n}=\left\{\left(x_{1}, \cdots, x_{n}\right) \in \mathbb{R}^{n} \mid x_{i} \geq 0, \forall i=1, \cdots, n\right\},
$$

then we have the following result:

Proposition 1 Let $\mathcal{C} \subset \mathbb{R}^{2(M+2)}$ be an integral curve of the system of ODEs given by the equations $(26 a)-(27 c)$ and suppose that at some time $t_{0}$ we have that $\mathcal{C}\left(t_{0}\right) \in$ $\mathbb{R}_{+}^{2(M+2)}$, then $\mathcal{C}(t) \in \mathbb{R}_{+}^{2(M+2)}$ for all $t \geq t_{0}$.

Proof We will assume any explicit time dependence through some or all of the parameters to be differentiable, thus the solutions to the equations will be defined over $\mathbb{R}$. For simplicity we will prove the statement restricted to the primary habitat given by the equations (26a)-(26c). Analogous arguments apply to the secondary habitat.

Let $P_{i}(t), P_{h}(t), P_{\dagger}(t)$ be a solution to the system of ODEs (26a)-(26c) and let $t_{0}$ be such that $P_{i}\left(t_{0}\right) \geq 0, P_{h}\left(t_{0}\right) \geq 0, P_{\dagger}\left(t_{0}\right) \geq 0$ for all $i \in\{1, \cdots, M\}$. Without loss of generality, $t_{0}$ can be taken as the initial time.

From the continuity of the solutions to a Lipschitz system of ODEs, as it is our case, it is clear that if $P_{1}, \cdots, P_{k}$ are all of the population densities being positive at $t_{0}$ all of them will remain positive throughout the interval

$\left(t_{0}-\epsilon_{+}, t_{0}+\epsilon_{+}\right)$, for some $\epsilon_{+}>0$. Accordingly, the positivity is critical only for those population densities vanishing at $t_{0}$.

Taking this into account and considering first the healthy population, if $P_{h}\left(t_{0}\right)=0$, the uniqueness theorem guarantees the existence of $\epsilon_{h}>0$ such that $P_{h}(t)=0, \forall t \in$ $\left(t_{0}-\epsilon_{h}, t_{0}+\epsilon_{h}\right)$.

Let us focus now on the malignant populations. Their evolution equations can be generically written as

$$
\dot{P}_{l}=f_{l} P_{l}+\sum_{j=1}^{M} \mathcal{M}_{l j} P_{j},
$$

with $f_{l}=\Gamma_{l}\left(\Psi-P_{h}-P_{\dagger}-\sum_{j=1}^{M} P_{j}\right)-\sum_{j=1}^{M} \mathcal{M}_{j l}-\Delta_{l}-k_{l}$.

Let $O_{0}=\left\{l_{1}, l_{2}, \cdots l_{k}\right\} \subset\{1, \cdots, M\}$ be the subset of malignant indices ${ }^{2}$ for which $P_{l_{j}}\left(t_{0}\right)=0$ and define $O_{1} \subseteq O_{0}$ as $O_{1}=\left\{i \in O_{0} \mid \forall j \notin O_{0}, \mathcal{M}_{i j}=0\right\}$. Thus, $\forall j \in O_{0} \backslash O_{1}$ it holds that $\dot{P}_{j}\left(t_{0}\right)>0$ while $\dot{P}_{l}\left(t_{0}\right)=P_{l}\left(t_{0}\right)=0$ for all $l \in O_{1}$. Now we have three possibilities depending on which set is $O_{1}$ :

1. $O_{1}=\emptyset$.

In that case the first derivative of every vanishing component is positive at $t_{0}$ and by the mean value theorem there will be $\epsilon_{\emptyset}>0$ such that $P_{i}(t)>0$ for all $t \in\left(t_{0}, t_{0}+\epsilon_{\emptyset}\right)$ and every $i \in O_{0}$.

\footnotetext{
2 We assume this set is not empty, otherwise there would be an open interval for which $P_{l}(t)>0$. If, instead, $O_{0}=\{1, \ldots, M\}$ then the solution would be the trivial one.
} 
2. $O_{1}=O_{0}$.

In that case, for all $l \in O_{0}, \dot{P}_{l}=f_{l} P_{l}+\sum_{k \in O_{0}} \mathcal{M}_{l k} P_{k}$ and hence the uniqueness theorem tells us that, for some $\epsilon_{e q}>0, \mathcal{P}_{i}(t)=0$ over $\left(t_{0}-\epsilon_{e q}, t_{0}+\epsilon_{e q}\right)$ for every $i \in O_{0}$.

3. $\varnothing \neq O_{1} \subset O_{0}$.

In that last case the mean value theorem guarantees that there is $\epsilon_{0}>1$ for which $P_{l}(t)>0$ over the interval $\left(t_{0}, t_{0}+\epsilon_{1}\right)$ and for every $l \in O_{0} \backslash O_{1}$.

In addition, since $\dot{P}_{l}\left(t_{0}\right)=P_{l}\left(t_{0}\right)=0$ for all $l \in O_{1}, \dot{P}_{s}\left(t_{0}\right)>0$ for any $s \in O_{0} \backslash O_{1}$ and, by definition, $\mathcal{M}_{i j}=0$ for every $i \in O_{1}$ and every $j \notin O_{0}$, we can start over the whole process by defining a new subset $O_{2}=$ $\left\{i \in O_{1} \mid \forall j \notin O_{1}, \mathcal{M}_{i j}=0\right\}$. Notice that for every $k \in O_{2}, \ddot{P}_{k}\left(t_{0}\right)=\dot{P}_{k}\left(t_{0}\right)=$ $P_{k}\left(t_{0}\right)=0$ while $\ddot{P}_{j}\left(t_{0}\right)>0$ for all $j \in O_{1} \backslash O_{2}$.

This iteration must come to an end since the original set is finite, meaning that there is $m \in \mathbb{N}$ for which $O_{m+1}$ is either empty or the same subset as $O_{m}$. Moreover, for every $1 \leq j \leq m$ and for every $l \in O_{j}$ it holds that $P_{l}\left(t_{0}\right)=\dot{P}_{l}\left(t_{0}\right)=\cdots=P_{l}^{(j)}\left(t_{0}\right)=0$ whereas for all $s \in O_{j} \backslash O_{j+1}$ we have $P_{s}^{(j+1)}\left(t_{0}\right)>0$. Using then the Taylor's theorem,

$$
P_{S}\left(t>t_{0}\right)=\frac{\left(t-t_{0}\right)^{j+1}}{(j+1) !} P_{S}^{(j+1)}(\tau), \quad \tau \in\left[t_{0}, t\right],
$$

we can assure that for $1 \leq j \leq m$ and for all $s \in O_{j-1} \backslash O_{j}$, there is $\epsilon_{j}>0$ for which $P_{s}(t)>0$ over $\left(t_{0}, t_{0}+\epsilon_{j}\right)$. Taking $\bar{\epsilon}=\min \left\{\epsilon_{1}, \ldots, \epsilon_{m}\right\}$ we have that for every $i \in O_{0} \backslash O_{m}, P_{i}(t)>0$ over $\left(t_{0}, t_{0}+\bar{\epsilon}\right)$, while for $O_{m}$, and according to the conditionals 1 and 2 above, there is $\tilde{\epsilon}>0$ such that $P_{r}(t) \geq 0$ over $\left(t_{0}, t_{0}+\tilde{\epsilon}\right)$ for all $r \in O_{m}$. Therefore, if $\epsilon^{\prime}=\min \left\{\bar{\epsilon}, \tilde{\epsilon}, \epsilon_{+}\right\}$then $P_{i}(t) \geq 0, P_{h}(t) \geq 0$ over $\left(t_{0}, t_{0}+\epsilon^{\prime}\right)$ and for all $i \in\{1, \cdots, M\}$.

Having that $P_{i}(t), P_{h}(t) \geq 0$ the non negativity of the necrotic population follows straightforwardly. Regarding the secondary tumor, similar arguments show the positivity of the solutions provided that $\tilde{k}_{i} \geq 0$.

Thus we can assure that for some $\epsilon>0$ all of the population densities will be non negative over $\left(t_{0}, t_{0}+\epsilon\right)$ and by the continuity of the solutions we have that those are again non negative at $t_{1}=t_{0}+\epsilon$. The same arguments show that the solutions will be again non negative over $\left[t_{0}, t_{0}+\epsilon+\epsilon_{1}\right]$ for some $\epsilon_{1}>0$ and hence the non negativity is guaranteed for any $t \geq t_{0}$.

\section{References}

Andasari V, Gerish A, Lolas G, South AP, Chaplain MAJ (2010) Mathematical modeling of cancer cell invasion of tissue: biological insight from mathematical analysis and computational simulation. J Math Biol 63:141-171

Baake E, Wagner H (2001) Mutation-selection models solved exactly with methods of statistical mechanics. Genet Res 78:93-117

Basanta D, Hatzikirou H, Deutsch A (2008) Studying the emergence of invasiveness in tumors using game theory. Eur Phys J B 63:393-397 
Bearer EL, Lowengrub JS, Frieboes HB, Chuang Y-L, Jin F, Wise SM, Ferrari M, Agus DB, Cristini V (2009) Multiparameter computational modeling of tumor invasion. Cancer Res 69:4493-4501

Bernards R, Weinberg RA (2002) Metastasis genes: a progression puzzle. Nature 418:823-823

Boushaba K, Levine HA, Nilsen-Hamilton M (2006) A mathematical model for the regulation of tumor dormancy based on enzyme kinetics. Bull Math Biol 68:1495-1526

Cairns RA, Harris IS, Mak TW (2011) Regulation of cancer cell metabolism. Nat Rev Cancer 11:85-95

Chaffer CL, Weinberg RA (2011) A perspective on cancer cell metastasis. Science 331:1559-1564 sites Nat Rev Cancer 2:563-572

Chambers A, Naumov G, Vantyghem S, Tuck A (2000) Molecular biology of breast cancer biology: clinical implications of experimental studies on metastatic inefficiency. Breast Can Res 2:400-407

Chambers A, Naumov GN, Varghese HJ, Nadkarni KV, MacDonald IC, Groom AC (2001) Critical steps in hematogenous metastasis: an overview. Surg Oncol Clin North Am 10:243-255

Chambers AF, Groom AC, MacDonald IC (2002) Dissemination and growth of cancer cells in metastatic sites. Nat Rev Cancer 2:563-572

Chen LL, Christakis NA, Barabási A-L, Deisboeck TS (2009) Cancer metastasis networks and the prediction of progression patterns. Br J Cancer 101:749-758

Coghlin C, Murray GI (2010) Current and emerging concepts in tumour metastasis. J Pathol 222:1-15

Cook LM, Hurst DR, Welch DR (2011) Metastasis suppressors and the tumor microenvironment. Semin Cancer Biol 21:113-122

Dattoli G, Guiot C, Delsanto PP, Ottaviani PL, Pagnutti S, Deisboeck TS (2009) Cancer metabolism and the dynamics of metastasis. J Theor Biol 256:305-310

Demicheli R, Retsky MW, Hrushesky WJM, Baum M, Gukas ID (2008) The effects of surgery on tumor growth: a century of investigations. Ann Oncol 19:1821-1828

Dingli D, Michor F, Antal T, Pacheco JM (2007) The emergence of tumor metastases. Cancer Biol Ther $6(3): 383-390$

DiSibio G, French SW (2008) Metastatic patterns of cancer: results from a large autopsy study. Arch Pathol Lab Med 132:931-939

Ebos JML, Lee CR, Cruz-Munoz W, Bjarnason GA, Christensen JG (2009) Accelerated metastasis after short-term treatment with a potent inhibitor of tumor angiogenesis. Cancer Cell 15:232-239

Eikenberry S, Thalhauser C, Kuang Y (2009) Tumor-immune interaction, surgical treatment and cancer recurrence in a mathematical model of melanoma. Plos Comput Biol 5:e1000362

Enderling H, Hlatky J, Hahnfeldt P (2009) Migration rules: tumours are conglomerates of self-metastases. Br J Cancer 100:1917-1925

Folkman J (2002) Role of angiogenesis in tumor growth and metastasis. Semin Oncol 29:15-18

Frieboes HB, Zheng X, Sun C-H, Tromberg B, Gatenby RA, Cristiny V (2006) An integrated computational/experimental model of tumor invasion. Cancer Res 66:1597-1604

Gatenby RA, Gawlinski ET (1996) A reaction-diffusion model of cancer invasion. Cancer Res 56: 5745-5753

Gatenby RJ, Gillies RJ (2004) Why do cancers have high aerobic glycolysis. Nat Rev Cancer 4:891-899

Gerisch A, Chaplain MAJ (2008) Mathematical modelling of cancer cell invasion of tissue: local and nonlocal models and the effect of adhesion. J Theor Biol 250:684-704

Haeno H, Michor F (2010) The evolution of tumor metastases during clonal expansion. J Theor Biol 263: 30-44

Hanahan D, Weinberg RA (2000) The hallmarks of cancer. Cell 100:57-70

Hanahan D, Weinberg RA (2011) The hallmarks of cancer: the next generation. Cell 144:646-674

Hanin L, Korosteleva O (2010) Does extirpation of the primary breast tumor give boost to growth of metastases? Evidence revealed by mathematical modeling. Math Biosci 223:133-141

Hayashida Y, Hirai T, Morishita S, Kitajima M, Murakami R, Korogi Y, Makino K, Nakamura H, Ikushima I, Yamura M, Kochi M, Kuratsu JI, Yamashita Y (2006) Diffusion-weighted imaging of metastatic brain tumors: comparison with histologic type and tumor cellularity. Am J Neuroradiol 27:1419-1425

Hess KR, Varadhachary GR, Taylor SH, Wei W, Raber MN, Lenzi R, Abbruzzese JL (2006) Metastatic patterns in adenocarcinoma. Cancer 106:1624-1633

Hüsemann Y, Geigl JB, Schubert F, Musiani P, Meyer M, Burghart E, Forni G, Eils R, Fehm T, Riethmüller G, CityKlein CA (2008) Systemic spread is an early step in breast cancer. Cancer Cell 13:58-68

Hurst DR, Welch DR (2011) Metastasis suppressor genes: at the interface between the environment and tumor cell growth. Int Rev Cell Mol Biol 286:107-180 
Iwasa Y, Michor F, Nowak MA (2004) Evolutionary dynamics of invasion and escape. J Theor Biol 226:205-214

Jain RK, Tong RT, Munn LL (2007) Effect of vascular normalization by antiangiogenic therapy on interstitial hypertension, peritumor edema and lymphatic metastasis: insights from a mathematical model. Cancer Res 67:2729-2735

Kendal WS (2001) The size distribution of human hematogenous metastases. J Theor Biol 211:29-38

Kim MY, Oskarsson T, Acharyya S, Nguyen DX, Zhang XHF, Norton L, Massagué J (2009) Tumor selfseeding by circulating cancer cells. Cell 139:1315-1326

Koppenol WH, Bounds PL, Dang CV (2011) Otto Warburg's contribution to current concepts of cancer metabolism. Nat Rev Cancer 11:325-337

Koscielny S, Tubiana M, Valleron A (1985) A simulation model of the natural history of human breast cancer. Br J cancer 52:515-524

Malanchi I, Santamaria-Martínez A, Susanto E, Peng H, Lehr HA, Delaloye JF, Huelsken J (2012) Interactions between cancer stem cells and their nicher govern metastatic colonization. Nature 481:85-89

Mendoza-Juez B, Martínez-González A, Calvo GF, Pérez-García VM (2011) A mathematical model for the glucose-lactate metabolism of in vitro cancer cells. Bull Math Biol. doi:10.1007/s11538-011-9711-z

Meng S et al (2004) Circulating tumor cells in patients with breast cancer dormancy. Clin Cancer Res 10:8152-8162

Michaelson JS, Cheongsiatmoy JA, Dewey F, Silverstein MJ, Sgroi D, Smith B, Tanabe KK (2005) Spread of human cancer cells occurs with probabilities indicative of a nongenetic mechanism. Br J Cancer 93:1244-1249

Michor F, Nowak MA, Iwasa Y (2006) Stochastic dynamics of metastasis formation. J Theor Biol 240: 521-530

Pantel K, Brakenhoff RH (2004) Dissecting the metastatic cascade. Nat Rev Cancer 4:448-456

Peeters CF, Westphal JR, de Waal RM, Ruiter DJ, Wobbes T, Ruers TJ (2004) Vascular density in colorectal liver metastases increases after removal of the primary tumor in human cancer patients. Int J Cancer 112:554-559

Peeters CFJM, Westphal JR, De Waal RMW, Ruiter DJ, Wobbes T, Oyen WJG, Ruers TJ (2005) Decrease in circulating anti-angiogenic factors (angiostatin and endostatin) after surgical removal of primary colorectal carcinoma coincides with increased metabolic activity of liver metastases. Surgery 137: 246-249

Peeters CF, de Waal RM, Wobbes T, Westphal JR, Ruers TJ (2006) Outgrowth of human liver metastases after resection of the primary colorectal tumor: a shift in the balance between apoptosis and proliferation. Int J Cancer 119:1249-1253

Peeters CF, de Waal RM, Ruiter DJ, Wobbes T, Ruers TJ (2008) Metastatic dormancy imposed by the primary tumor: does it exist in humans. Ann Surg Oncol 15:3308-3315

Podsypanina K, Du Y-C N, Jechlinger M, Beverly LJ, Hambardzumyan D, Varmus H (2008) Seeding and propagation of untransformed mouse mammary cells in the lung. Science 321:1841-1844

Ramis-Conde I, Chaplain MAJ, Anderson ARA (2008) Mathematical modelling of cancer cell invasion of tissue. Math Comput Model 47:533-545

Retsky MW, Demicheli R, Swartzendruber DE, Bame PD, Wardwell RH, Bonadonna G, Speer JF, Valagussa P (1997) Computer simulation of a breast cancer metastasis model. Breast Cancer Res Treat 45:193-202

Smallbone K, Gatenby RA, Maini PK (2008) Mathematical modelling of tumour acidity. J Theor Biol 255:106-112

Sonveaux P, Végran F, Schroeder T, Wergin MC, Verrax J, Rabbani ZN, de Saedeleer CJ, Kennedy KM, Diepart C, Jordan BF, Kelley MJ, Gallez B, Wahl ML, Feron O, Dewhirst MW (2008) Targeting lactate-fueled respiration selectively kills hypoxic tumor cells in mice. J Clin Invest 118:3930-3942

Traulsen A, Nowak MA, Pacheco JM (2006) Stochastic dynamics of invasion and fixation. Phys Rev E 74:011909

Vander-Heiden MG, Locasale JW, Swanson KD, Sharfi H, Heffron GJ, Amador-Noguez D, Christofk HR, Wagner G, Rabinowitz JD, Asara JM, Cantley LC (2010) Evidence for an alternative glycolytic pathway in rapidly proliferating cells. Science 329:1492-1499

Waclaw R, Allen RJ, Evans R (2010) Dynamical phase transition in a model for evolution with migration. Phys Rev Lett 105:268101

Warburg O (1956) On the origin of cancer cells. Science 123:309-314

Warburg O, Posener K, Negelein E (1924) On the metabolism of carcinoma cells. Biochem Z 152:309-344 
Weinberg RA (2008) Leaving home early: re-examination of the canonical models of tumour progression. Cancer Cell 14:283-284

Weiss L (2000) Metastasis of cancer: a conceptual history from antiquity to the 1990s. Cancer Metastasis Rev 19:193-383

Wilson RW, Hay PH (2011) Targeting hypoxia in cancer therapy. Nat Rev Cancer 11:393-410

Yorke ED, Fuks Z, Norton L, Whitmore W, Ling CC (1993) Modeling the development of metastases from primary and locally recurrent tumors: comparison with a clinical data base for prostatic cancer. Cancer Res 53:2987-2993 